\title{
OPE and a low-energy theorem in QCD-like theories
}

\author{
Matteo Becchetti ${ }^{a, b}$ and Marco Bochicchio ${ }^{b}$ \\ ${ }^{a}$ Dipartimento di Fisica, Sapienza - Università di Roma, \\ Piazzale Aldo Moro 5, 00185, Rome, Italy \\ ${ }^{b}$ INFN Sezione di Roma, \\ Piazzale Aldo Moro 2, 00185, Rome, Italy \\ E-mail: matteo.becchetti@roma1.infn.it, \\ marco.bochicchio@roma1.infn.it
}

ABSTRACT: We verify, both perturbatively and nonperturbatively asymptotically in the ultraviolet (UV), a special case of a low-energy theorem of the NSVZ type in QCD-like theories, recently derived in Phys. Rev. D 95 (2017) 054010, that relates the logarithmic derivative with respect to the gauge coupling, or the logarithmic derivative with respect to the renormalization-group (RG) invariant scale, of an $n$-point correlator of local operators in one side to an $n+1$-point correlator with the insertion of $\operatorname{Tr} F^{2}$ at zero momentum in the other side. Our computation involves the operator product expansion (OPE) of the scalar glueball operator, $\operatorname{Tr} F^{2}$, in massless QCD, worked out perturbatively in JHEP 12 (2012) 119 - and in its RG-improved form in the present paper — by means of which we extract both the perturbative divergences and the nonperturbative UV asymptotics in both sides. We also discuss the role of the contact terms in the OPE, both finite and divergent, discovered some years ago in JHEP 12 (2012) 119, in relation to the low-energy theorem. Besides, working the other way around by assuming the low-energy theorem for any 2-point correlator of a multiplicatively renormalizable gauge-invariant operator, we compute in a massless QCD-like theory the corresponding perturbative OPE to the order of $g^{2}$ and nonperturbative asymptotics. The low-energy theorem has a number of applications: to the renormalization in asymptotically free QCD-like theories, both perturbatively and nonperturbatively in the large- $N$ 't Hooft and Veneziano expansions, and to the way the open/closed string duality may or may not be realized in the would-be solution by canonical string theories for QCD-like theories, both perturbatively and in the 't Hooft large- $N$ expansion. Our computations will also enter further developments based on the low-energy theorem.

KEYWords: Perturbative QCD, Renormalization Group

ArXiv EPrint: 1810.08527 


\section{Contents}

1 Introduction $\quad 1$

1.1 Physics motivations 1

1.2 Plan of the paper 2

2 The low-energy theorem 3

2.1 Low-energy theorem in terms of the Wilsonian coupling 3

2.2 Low-energy theorem in terms of $\Lambda_{\mathrm{QCD}} \quad 4$

2.3 Low-energy theorem in terms of the canonical coupling 4

3 Main technical arguments $\quad \mathbf{5}$

3.1 Verifying eq. (1.3) by the perturbative OPE 5

3.2 Perturbative OPE for $F^{2}(x) F^{2}(0) \quad 7$

3.3 Verifying eq. (1.3) for $\left\langle F^{2}(z) F^{2}(0)\right\rangle \quad 9$

3.4 Verifying eq. (1.4) by the RG-improved OPE 9

4 Low-energy theorem for $\left\langle F^{2}(z) F^{2}(0)\right\rangle$ in perturbation theory $\quad 10$

4.1 Order of $g^{2} \quad 11$

$\begin{array}{lll}4.2 & \text { Order of } g^{4} & 12\end{array}$

5 Nonperturbative UV asymptotics of the low-energy theorem for $\begin{array}{ll}\left\langle F^{2}(z) F^{2}(0)\right\rangle & 14\end{array}$

6 Perturbative OPE to the order of $g^{2}$ and nonperturbative UV asymptotics from the low-energy theorem for $\langle O(z) O(0)\rangle \quad 16$

7 Conclusions $\quad 19$

$\begin{array}{lll}7.1 & \text { Low-energy theorem and perturbation theory } & 19\end{array}$

7.2 Low-energy theorem and nonperturbative UV asymptotics 20

$\begin{array}{ll}\text { A Nonperturbative UV asymptotics of the OPE } & 21\end{array}$

B Perturbative OPE 23

B.1 Perturbative $C_{0}^{(S)}$ in the momentum representation 23

B.2 Perturbative $C_{0}^{(S)}$ in the coordinate representation 25

B.3 Verifying the UV asymptotics of $C_{0}^{(S)}$ by a change of renormalization scheme $\begin{array}{ll}\text { in perturbation theory } & 27\end{array}$

B.4 Perturbative $C_{1}^{(S)}$ in the momentum representation 29

B.5 Perturbative $C_{1}^{(S)}$ in the coordinate representation 31

B.6 Verifying the UV asymptotics of $C_{1}^{(S)}$ by a change of renormalization scheme in perturbation theory 


\section{Introduction}

\subsection{Physics motivations}

One of the aims of the present paper is to verify, both perturbatively and nonperturbatively asymptotically in the ultraviolet (UV), a special case of a recently derived low-energy theorem [1] of the Novikov-Shifman-Vainshtein-Zakharov (NSVZ) type [2] in SU(N) QCDlike gauge theories.

It relates the logarithmic derivative with respect to the 't Hooft gauge coupling, $g^{2}=$ $g_{Y M}^{2} N$, of an $n$-point correlator of local operators, $\mathcal{O}_{k}$, to an $n+1$-point correlator with the insertion of $\operatorname{Tr} \mathcal{F}^{2}$ at zero momentum [1]:

$$
\frac{\partial\left\langle\mathcal{O}_{1} \cdots \mathcal{O}_{n}\right\rangle}{\partial \log g}=\frac{N}{g^{2}} \int\left\langle\mathcal{O}_{1} \cdots \mathcal{O}_{n} \operatorname{Tr} \mathcal{F}^{2}(x)\right\rangle-\left\langle\mathcal{O}_{1} \cdots \mathcal{O}_{n}\right\rangle\left\langle\operatorname{Tr} \mathcal{F}^{2}(x)\right\rangle d^{4} x
$$

where the Wilsonian normalization of the Yang-Mills (YM) action is chosen (subsection 2.1) and the operators, $\operatorname{Tr} \mathcal{F}^{2}$ and $\mathcal{O}_{k}$, are $g$ independent (subsection 2.1).

It admits another version [1], where the logarithmic derivative with respect to the gauge coupling is replaced by the logarithmic derivative with respect to the renormalizationgroup (RG) invariant scale, $\Lambda_{\mathrm{QCD}}$, in an asymptotically free (AF) QCD-like theory (subsection 2.2):

$$
\frac{\partial\left\langle\mathcal{O}_{1} \ldots \mathcal{O}_{n}\right\rangle}{\partial \log \Lambda_{\mathrm{QCD}}}=-\frac{N \beta(g)}{g^{3}} \int\left\langle\mathcal{O}_{1} \ldots \mathcal{O}_{n} \operatorname{Tr} \mathcal{F}^{2}(x)\right\rangle-\left\langle\mathcal{O}_{1} \ldots \mathcal{O}_{n}\right\rangle\left\langle\operatorname{Tr} \mathcal{F}^{2}(x)\right\rangle d^{4} x
$$

After rescaling the gauge fields in the functional integral by a factor of $\frac{g}{\sqrt{N}}$, the low-energy theorem admits a trivially equivalent canonical version with the canonical normalization of the YM action (subsection 2.3):

$$
\begin{aligned}
& \left(\sum_{k=1}^{k=n} c_{k}\right)\left\langle O_{1} \ldots O_{n}\right\rangle+\frac{\partial\left\langle O_{1} \ldots O_{n}\right\rangle}{\partial \log g} \\
& \quad=\int\left\langle O_{1} \ldots O_{n} \operatorname{Tr} F^{2}(x)\right\rangle-\left\langle O_{1} \ldots O_{n}\right\rangle\left\langle\operatorname{Tr} F^{2}(x)\right\rangle d^{4} x
\end{aligned}
$$

in terms of operators, $\operatorname{Tr} F^{2}$ and $O_{k}$, defined by the very same rescaling, satisfying $\frac{g^{2}}{N} \operatorname{Tr} F^{2}=\operatorname{Tr} \mathcal{F}^{2}$ and $\left(\frac{g}{\sqrt{N}}\right)^{c_{k}} O_{k}=\mathcal{O}_{k}$ for some $c_{k}$. The canonically normalized operators, $\operatorname{Tr} F^{2}$ and $O_{k}$, depend now on $g$ (subsection 2.3). Eq. (1.2) also admits a canonical version (subsection 2.3):

$$
\begin{aligned}
- & \frac{\beta(g)}{g}\left(\sum_{k=1}^{k=n} c_{k}\right)\left\langle O_{1} \ldots O_{n}\right\rangle+\frac{\partial\left\langle O_{1} \ldots O_{n}\right\rangle}{\partial \log \Lambda_{\mathrm{QCD}}} \\
& =-\frac{\beta(g)}{g} \int\left\langle O_{1} \ldots O_{n} \operatorname{Tr} F^{2}(x)\right\rangle-\left\langle O_{1} \ldots O_{n}\right\rangle\left\langle\operatorname{Tr} F^{2}(x)\right\rangle d^{4} x
\end{aligned}
$$

The canonical version of eq. (1.1), which is most suitable for perturbative computations, - eq. (1.3) for $n=2$ and $O_{k}=\operatorname{Tr} F^{2}$ — has been employed to analyze the renormalization properties of QCD-like theories perturbatively to the order of $g^{2}$ in [3] and the way the 
open/closed string duality $[4,5]$ may be actually implemented [3] in string theories realizing perturbatively [5] QCD-like theories.

The second version - eq. (1.2) with $\mathcal{O}_{k}=\operatorname{Tr} \mathcal{F}^{2}$ — has been employed to compute the nonperturbative countertems [1] in the large- $N$ 't Hooft [6] and Veneziano [7] expansions of QCD-like theories.

Moreover, it has entered crucially a no-go theorem [3] that the nonperturbative renormalization in the 't Hooft large- $N$ QCD S matrix is incompatible with the open/closed string duality of a would-be canonical string solution, which therefore does not exist [3].

By a canonical string solution we mean [3] a perturbative expansion in the string coupling, $g_{s} \sim \frac{1}{N}$, for the string $\mathrm{S}$ matrix that matches the topology of the 't Hooft large- $N$ expansion and computes the large- $N$ QCD S matrix by means of an auxiliary $2 \mathrm{~d}$ conformal field theory living on the string world-sheet with fixed topology [6-9].

A noncanonical way-out to the no-go theorem has been suggested in $[3,10]$. Relatedly, the large- $N$ Veneziano expansion [7] has been discussed in $[1,3,10]$.

Because of the importance of the low-energy theorem for the above subjects, a deeper understanding and an explicit evaluation of both sides in eqs. (1.3) and (1.4) are most interesting.

\subsection{Plan of the paper}

In section 2 we recall the proof of various versions of the low-energy theorem in [1].

In section 3 we describe the rationale behind our computations based on the operator product expansion (OPE) in relation to the low-energy theorem.

In subsection 3.2 we summarize the result of the computation in [11-13], which we employ in the present paper, of the perturbative OPE coefficients for $\operatorname{Tr} F^{2}(x) \operatorname{Tr} F^{2}(0)$ (eqs. (3.4), (3.5) and (3.6)) in QCD with massless quarks (massless QCD for short).

In section 4 we verify the low-energy theorem perturbatively to the order of $g^{4}$ for $n=2$ and $O_{k}=\operatorname{Tr} F^{2}$ on the basis of the aforementioned OPE in [11-13].

In fact, the divergent parts in both sides of eq. (1.3) for $n=2$ and $O_{k}=\operatorname{Tr} F^{2}$ have already been computed to the order of $g^{2}$ in perturbation theory in [3], thus partially verifying a special case of the low-energy theorem perturbatively.

Yet, we include (subsection 4.1) in the aforementioned computation the finite contact term to the order of $g^{0}$ in $C_{1}^{(S)}$ (eq. (3.6)) [11], which arises from performing the OPE in eq. (1.3), that has been skipped in [3].

Moreover, we extend the perturbative computation in [3] to the order of $g^{4}$ in subsection 4.2, including as well the divergent contact term to the order of $g^{4}$ in $C_{1}^{(S)}$ (eq. (3.6)) discovered some years ago in [11], whose renormalization has been recently discussed in [13].

In section 5 we compute the nonperturbative universal, i.e., renormalization-scheme independent, UV asymptotics ${ }^{1}$ in the coordinate representation in both sides of eq. (1.4) for $n=2$ and $O_{k}=\operatorname{Tr} F^{2}$.

Our nonperturbative computation furnishes a detailed derivation and an improvement to the next to leading logs of the crucial — for the no-go theorem in [3] — nonperturbative

\footnotetext{
${ }^{1}$ We define in appendix A what we mean by the universal UV asymptotics.
} 
UV leading-log asymptotic estimate in the coordinate representation in [3], and provides another check of the low-energy theorem.

In order to perform the computation, we employ firstly the perturbative OPE of $\operatorname{Tr} F^{2}(x) \operatorname{Tr} F^{2}(0)$ worked out in [11-13] that is recalled in appendix B. A previous perturbative lower-order computation appeared in [14]. We pass from the perturbative OPE in the momentum representation [11-13] in appendices B.1 and B.4 to the OPE in the coordinate representation by the Fourier transform in appendices B.2 and B.5. From it we get the normalization for the nonperturbative universal RG-improved UV asymptotics of the OPE coefficients in the coordinate representation derived a priori in appendix A, which includes the leading and next to leading logs.

Moreover, by making the OPE coefficients RG invariant by a suitable rescaling of the operators, we verify in appendices B.3 and B.6 their nonperturbative UV asymptotics by rewriting the perturbative computation, originally expressed in terms of $g(\mu)$ and large logs [11-13], in terms of the running coupling, $g(x)$, to the given perturbative order.

In passing, for future applications, we compute in appendix B.3 the nonperturbative UV asymptotics in massless QCD of the 2-point correlator of $\frac{g^{2}}{N} \operatorname{Tr} F^{2}-$ the YM Lagrangian density with the canonical normalization (subsection 2.3) - which coincides with the continuum limit of the 2-point correlator of the Wilson plaquette on the lattice (subsection 2.3). As the operator $\frac{g^{2}}{N} \operatorname{Tr} F^{2}$ is not RG invariant, our computation also includes the scale-dependent corrections to the universal UV asymptotics (appendix B.3).

Previous related results about the universal asymptotics of the OPE both in the momentum and coordinate representation have been obtained in $[15,16]$ and, only about the leading logs, in the momentum representation in [17] and in the coordinate representation in [3].

In section 6, working the other way around by assuming the low-energy theorem for any 2-point correlator, $\langle O(z) O(0)\rangle$, of a canonically normalized multiplicatively renormalizable gauge-invariant operator $O$, we compute the corresponding perturbative OPE to the order of $g^{2}$ and nonperturbative UV asymptotics.

In section 7 we summarize our conclusions. Our computations will also enter further developments that assume the low-energy theorem.

\section{The low-energy theorem}

\subsection{Low-energy theorem in terms of the Wilsonian coupling}

For completeness we report the proof of the low-energy theorem in QCD-like theories according to [1].

Given a set of local operators, $\mathcal{O}_{k}$, and the Wilsonian normalization of the YM action, by deriving:

$$
\left\langle\mathcal{O}_{1} \cdots \mathcal{O}_{n}\right\rangle=\frac{\int \mathcal{O}_{1} \cdots \mathcal{O}_{n} e^{-\frac{N}{2 g^{2}} \int \operatorname{Tr} \mathcal{F}^{2}(x) d^{4} x+\cdots}}{e^{-\frac{N}{2 g^{2}} \int \operatorname{Tr} \mathcal{F}^{2}(x) d^{4} x+\cdots}}
$$


with respect to $-\frac{1}{g^{2}}$, we obtain:

$$
\frac{\partial\left\langle\mathcal{O}_{1} \cdots \mathcal{O}_{n}\right\rangle}{\partial \log g}=\frac{N}{g^{2}} \int\left\langle\mathcal{O}_{1} \cdots \mathcal{O}_{n} \operatorname{Tr} \mathcal{F}^{2}(x)\right\rangle-\left\langle\mathcal{O}_{1} \cdots \mathcal{O}_{n}\right\rangle\left\langle\operatorname{Tr} \mathcal{F}^{2}(x)\right\rangle d^{4} x
$$

where the trace, $\operatorname{Tr}$, is in the fundamental representation, $\operatorname{Tr} \mathcal{F}^{2} \equiv \operatorname{Tr}\left(\mathcal{F}_{\mu \nu} \mathcal{F}^{\mu \nu}\right)$, the sum over repeated indices is understood, and $\mathcal{F}_{\mu \nu}=\partial_{\mu} A_{\nu}-\partial_{\nu} A_{\mu}+i\left[A_{\mu}, A_{\nu}\right]$.

From the derivation it is clear that all the operators $-\mathcal{O}_{k}, \operatorname{Tr} \mathcal{F}^{2}$, and in the dots are chosen to be $g$ independent. It also is clear that $g$ in eqs. (2.1) and (2.2) is the bare coupling.

Interestingly, the Wilsonian normalization of the YM action in eq. (2.1) also occurs in nonperturbative computations in lattice gauge theories.

\subsection{Low-energy theorem in terms of $\Lambda_{\mathrm{QCD}}$}

A second version [1] of the low-energy theorem holds in an AF QCD-like theory:

$$
\frac{\partial\left\langle\mathcal{O}_{1} \ldots \mathcal{O}_{n}\right\rangle}{\partial \log \Lambda_{\mathrm{QCD}}}=-\frac{N \beta(g)}{g^{3}} \int\left\langle\mathcal{O}_{1} \ldots \mathcal{O}_{n} \operatorname{Tr} \mathcal{F}^{2}(x)\right\rangle-\left\langle\mathcal{O}_{1} \ldots \mathcal{O}_{n}\right\rangle\left\langle\operatorname{Tr} \mathcal{F}^{2}(x)\right\rangle d^{4} x
$$

as it follows by employing the chain rule, $\frac{\partial}{\partial \log g}=\frac{\partial \Lambda_{\mathrm{QCD}}}{\partial \log g} \frac{\partial}{\partial \Lambda_{\mathrm{QCD}}}$, the defining relation:

$$
\left(\frac{\partial}{\partial \log \Lambda}+\beta(g) \frac{\partial}{\partial g}\right) \Lambda_{\mathrm{QCD}}=0
$$

and the identity:

$$
\frac{\partial \Lambda_{\mathrm{QCD}}}{\partial \log \Lambda}=\Lambda_{\mathrm{QCD}}
$$

since $\Lambda_{\mathrm{QCD}}=e^{\log \Lambda} f(g)$ for a function $f(g)$.

\subsection{Low-energy theorem in terms of the canonical coupling}

In order to verify the low-energy theorem in perturbation theory it is convenient to employ the canonical normalization of the YM action [3].

Thus, we rescale the gauge fields in the functional integral by a factor of $\frac{g}{\sqrt{N}}$. Of course, the rescaling does not affect the vev of the operators, as it is just a change of variables. ${ }^{2}$

Therefore, defining after the rescaling, $\frac{g^{2}}{N} \operatorname{Tr} F^{2}=\operatorname{Tr} \mathcal{F}^{2}$ and $\left(\frac{g}{\sqrt{N}}\right)^{c_{k}} O_{k}=\mathcal{O}_{k}$ for some $c_{k}$, where now $F_{\mu \nu}=\partial_{\mu} A_{\nu}-\partial_{\nu} A_{\mu}+i \frac{g}{\sqrt{N}}\left[A_{\mu}, A_{\nu}\right]$ and $O_{k}$ are $g$ dependent but canonically normalized, we obtain the identity:

$$
\left\langle\mathcal{O}_{1} \ldots \mathcal{O}_{n}\right\rangle=\prod_{k=1}^{k=n}\left(\frac{g}{\sqrt{N}}\right)^{c_{k}}\left\langle O_{1} \ldots O_{n}\right\rangle
$$

\footnotetext{
${ }^{2}$ Certainly, this statement holds to every order of perturbation theory. It also holds nonperturbatively provided that the theory is regularized, as for example in dimensional regularization, in such a way that no rescaling anomaly arises [18]. Moreover, it holds nonperturbatively as well if the theory is regularized on a finite lattice, since then the functional integral is finite dimensional and no rescaling anomaly may arise.
} 
where the vev in the l.h.s. is defined with the Wilsonian normalization in eq. (2.1) and in the r.h.s. with the canonical normalization, i.e., after the aforementioned rescaling of the gauge fields in the functional integral in eq. (2.1).

Interestingly, for $\operatorname{Tr} \mathcal{F}^{2}$ - the YM Lagrangian density with the Wilsonian normalization, which coincides with the continuum limit of the Wilson plaquette on the lattice eq. (2.6) reduces to:

$$
\left\langle\operatorname{Tr} \mathcal{F}^{2} \cdots \operatorname{Tr} \mathcal{F}^{2}\right\rangle=\left\langle\frac{g^{2}}{N} \operatorname{Tr} F^{2} \cdots \frac{g^{2}}{N} \operatorname{Tr} F^{2}\right\rangle
$$

since the Wilsonian normalization of the YM action occurs in lattice gauge theories as well (subsection 2.1).

As a consequence, after the rescaling, eq. (2.2) reads:

$$
\begin{aligned}
& \left(\sum_{k=1}^{k=n} c_{k}\right)\left\langle O_{1} \ldots O_{n}\right\rangle+\frac{\partial\left\langle O_{1} \ldots O_{n}\right\rangle}{\partial \log g} \\
& \quad=\int\left\langle O_{1} \ldots O_{n} \operatorname{Tr} F^{2}(x)\right\rangle-\left\langle O_{1} \ldots O_{n}\right\rangle\left\langle\operatorname{Tr} F^{2}(x)\right\rangle d^{4} x
\end{aligned}
$$

Similarly, eq. (2.3) becomes:

$$
\begin{aligned}
- & \frac{\beta(g)}{g}\left(\sum_{k=1}^{k=n} c_{k}\right)\left\langle O_{1} \ldots O_{n}\right\rangle+\frac{\partial\left\langle O_{1} \ldots O_{n}\right\rangle}{\partial \log \Lambda_{\mathrm{QCD}}} \\
= & -\frac{\beta(g)}{g} \int\left\langle O_{1} \ldots O_{n} \operatorname{Tr} F^{2}(x)\right\rangle-\left\langle O_{1} \ldots O_{n}\right\rangle\left\langle\operatorname{Tr} F^{2}(x)\right\rangle d^{4} x
\end{aligned}
$$

\section{Main technical arguments}

\subsection{Verifying eq. (1.3) by the perturbative OPE}

Eqs. (1.3) and (2.8) apply to any product, $O_{1} \ldots O_{n}$, of bare - canonically normalized local operators, not necessarily gauge invariant.

In order to verify perturbatively eq. (1.3), we assume that such local operators have a well defined - in general nonvanishing - anomalous dimension (section 6).

Moreover, for the explicit computations in the present paper, we restrict in sections 4,5 and 6 to gauge-invariant operators.

In gauge-fixed perturbation theory and in renormalization schemes that preserve the BRST invariance, gauge-invariant operators, which after gauge-fixing become BRTS invariant, may mix [19-21] under renormalization only with themselves, with operators that are BRTS exact, and with operators that vanish by the equations of motion. The BRST-exact operators may mix [19-21] only with themselves and with operators that vanish by the equations of motion. The latter may mix [19-21] only with themselves. Thus, the mixing matrix for gauge-invariant operators has a triangular structure [19-21] that simplifies the computation of the anomalous dimensions. 
For example, $\operatorname{Tr} F^{2}$ mixes in QCD with certain dimension-4 BRST-exact operators [22], and with the gauge-invariant operators, $m \bar{\psi} \psi$ and $\bar{\psi}(\not D+m) \psi{ }^{3}$ [22]. The latter vanishes by the equations of motion.

Now, the correlators of BRST-exact operators with gauge-invariant operators vanish [19-21]. Moreover, the insertion in the vev of operators that vanish by the equations of motion may only produce contact terms [23]. Therefore, by limiting ourselves to correlators of gauge-invariant operators, the mixing of gauge-invariant operators with the aforementioned ones may be safely ignored.

For computational reasons, we specialize from now on to a QCD-like theory massless in perturbation theory - a massless QCD-like theory for short - .

In a massless QCD-like theory there is no mixing of $\operatorname{Tr} F^{2}$ with operators containing a mass parameter - like $m \bar{\psi} \psi$ - since they vanish. Besides, according to the above discussion, the mixing of $\operatorname{Tr} F^{2}$ with the remaining dimension- 4 operators may be ignored - up to, perhaps, contact terms - in gauge-invariant correlators.

Therefore, as far as the computations in the present paper are concerned, we may consider $\operatorname{Tr} F^{2}$ to be multiplicatively renormalizable with a well-defined anomalous dimension.

Moreover, restricting to a massless QCD-like theory is specifically convenient because a vast family of canonically normalized gauge-invariant operators exists whose correlators are conformal invariant to the order of $g^{2}$ [24]. This holds for primary conformal operators to the order $g^{2}$ [24] in the conformal renormalization scheme [24].

Indeed, the conformal symmetry is manifest to the order of $g^{2}$ in a massless QCDlike theory, since the beta function only affects the solution of the Callan-Symanzik (CS) equation starting from the order of $g^{4}[24,25]$.

Besides, due to the conformal symmetry, provided that the mixing matrix can be diagonalized, ${ }^{4}$ an orthogonal basis of canonically normalized primary conformal operators exists, such that the mixed 2-point correlators, $\left\langle O_{i}(x) O_{k}(0)\right\rangle$, vanish for $i \neq k$ to the order of $g^{2}$, as we show momentarily.

For primary conformal operators, $O_{i}$, with different conformal dimensions to the order of $g^{2}, \Delta_{i}=D_{i}-\gamma_{0}^{\left(O_{i}\right)} g^{2}$, with $\gamma_{0}^{\left(O_{i}\right)}$ the first coefficient of the anomalous dimension defined in eq. (4.2), the orthogonality is a consequence of the conformal symmetry. For primary conformal operators of spin $s$ with the same conformal dimension, the 2-point mixed correlator in a generic operator basis reads for $x \neq 0$ in the conformal scheme to the order of $g^{2}$ :

$$
G_{i k}^{(2)}(x)=\left\langle O_{i}(x) O_{k}(0)\right\rangle=A_{i k}^{(s)}(g) \frac{P^{(s)}(x)}{x^{2 D}}\left(1+g^{2}(\mu) \gamma_{0} \log \left(x^{2} \mu^{2}\right)\right)
$$

with $P^{(s)}(x)$ the spin projector in the coordinate representation in the conformal limit, $A_{i k}^{(s)}(g)$ a constant matrix, $D$ the canonical dimension, and $\gamma_{0}$ the common first coefficient of the anomalous dimension of the operators. Thus, since for gauge-invariant operators that satisfy the spin-statistics theorem the matrix $A_{i k}^{(s)}(g)$ is symmetric, it can always be

\footnotetext{
${ }^{3}$ We employ the Euclidean notation.

${ }^{4}$ The precise conditions are worked out in [26].
} 
diagonalized by a change of the operator basis ${ }^{5}$ [25]. The existence of the orthogonal basis to the order of $g^{2}$ is employed in section 6 .

For the above operators the l.h.s. of eq. (1.3) is UV log divergent in perturbation theory to the order of $g^{2}$ because of the nontrivial anomalous dimensions of the operators.

The UV log divergences in the l.h.s. must be reproduced in the r.h.s. by the spacetime integration as the operator $\operatorname{Tr} F^{2}$ gets close to each $O_{k}$. Hence, we may evaluate the divergent parts in the r.h.s. by the OPE.

We specialize now to the case $n=2$ and $O_{k}=O_{1}$ for $k=1,2$ (sections 4 and 6).

In this case, we show momentarily that the leading perturbative UV log divergences in the r.h.s. of eq. (1.3) arise from the space-time integration of the following OPE (appendix A):

$$
\operatorname{Tr} F^{2}(x) O_{1}(y) \sim C_{O_{1}}^{\left(\operatorname{Tr} F^{2}, O_{1}\right)}(x-y) O_{1}(y)+\cdots
$$

where the dots include operators different from $O_{1}$.

Indeed, in order to produce, after the space-time integration, the desired UV log divergence, the coefficient $C_{O_{1}}^{\left(\operatorname{Tr} F^{2}, O_{1}\right)}$ must have canonical dimension 4 .

Thus, given that $\operatorname{Tr} F^{2}$ has canonical dimension 4, only the contribution of the operators $O_{k}$ with the same canonical dimension as $O_{1}$, may lead via the OPE, after the space-time integration in the r.h.s. of eq. (1.3), to the desired UV log divergence:

$$
\operatorname{Tr} F^{2}(x) O_{i}(y) \sim \sum_{k} C_{O_{k}}^{\left(\operatorname{Tr} F^{2}, O_{i}\right)}(x-y) O_{k}(y)+\cdots
$$

Higher-dimension operators in the OPE furnish UV finite contributions in the rhs, while lower-dimension operators furnish potential power-like divergences that, however, are absent in the 1.h.s. of eq. (1.3) in dimensional regularization.

Moreover, for $n=2$, we show in section 6 that, due to the assumed orthogonality of the operators $O_{k}$ to the order of $g^{2}$, the leading contribution in perturbation theory to the 3-point correlator in the r.h.s. of eq. (1.3) arising from the coefficients of dimension 4 occurs from the coefficient of the operator $O_{1}$ itself.

Therefore, by employing the OPE in eq. (3.2), we should be able to verify the equality of the universal, i.e., scheme-independent, divergent parts in both sides of eq. (1.3).

Indeed, our computation cannot be exact, but it is affected by finite ambiguities due both to the incomplete OPE and to regularizing in the infrared (IR) the integral in the r.h.s. of eq. (1.3) in perturbation theory (section 4).

\subsection{Perturbative OPE for $F^{2}(x) F^{2}(0)$}

In order to verify the above statements, the relevant OPE must be known explicitly. This is the case for the operator $O_{1}=\operatorname{Tr} F^{2}$, thanks to the results in [11-13] recalled here below.

For brevity we define $F^{2}(x) \equiv 2 \operatorname{Tr} F^{2}(x)$. The perturbative OPE for $F^{2}(x) F^{2}(0)$ in massless QCD reads:

$$
F^{2}(x) F^{2}(0)=C_{0}^{(S)}(x) \mathbb{I}+C_{1}^{(S)}(x) F^{2}(0)+\cdots
$$

\footnotetext{
${ }^{5}$ For a change of the operator basis, $O^{\prime}=S O$ in matrix notation, $G^{(2)}$ transforms as $G^{(2)^{\prime}}=S G^{(2)} S^{T}$, with $S^{T}$ the transposed of the matrix $S$ [26]. Therefore, a suitable transformation can always diagonalize the - possibly complex - symmetric matrix $A^{(s)}(g)$.
} 
$C_{0}^{(S)}(x)$ is the coefficient of the identity operator, $\mathbb{I}, C_{1}^{(S)}(x)$ is the coefficient of the operator $F^{2}$ itself and the dots stand for other operators that are irrelevant for the purpose of checking the universal log-divergent parts in both sides of eq. (1.3) according to the above discussion.

For multiplicatively renormalized $F^{2}$ in massless $\mathrm{QCD}, C_{0}^{(S)}$ has been computed perturbatively in the $\overline{M S}$ scheme to three loops, both in the momentum and coordinate representation, in [11]:

$$
\begin{aligned}
C_{0}^{(S)}(x)= & \frac{N^{2}-1}{x^{8}} \frac{48}{\pi^{4}}\left(1+g^{2}(\mu)\left(A_{0,1}^{(S)}+2 \beta_{0} \log \left(x^{2} \mu^{2}\right)\right)\right. \\
& \left.+g^{4}(\mu)\left(A_{0,2}^{(S)}+A_{0,3}^{(S)} \log \left(x^{2} \mu^{2}\right)+3 \beta_{0}^{2} \log ^{2}\left(x^{2} \mu^{2}\right)\right)\right) \\
+ & \Delta^{2} \delta^{(4)}(x) \frac{N^{2}-1}{4 \pi^{2}}\left(1+\log \left(\frac{\Lambda^{2}}{\mu^{2}}\right)+g^{2}(\mu)\left(A_{0,4}^{(S)}+A_{0,5}^{(S)} \log \left(\frac{\Lambda^{2}}{\mu^{2}}\right)\right.\right. \\
& \left.-\beta_{0} \log ^{2}\left(\frac{\Lambda^{2}}{\mu^{2}}\right)\right)+g^{4}(\mu)\left(A_{0,6}^{(S)}+A_{0,7}^{(S)} \log \left(\frac{\Lambda^{2}}{\mu^{2}}\right)+A_{0,8}^{(S)} \log ^{2}\left(\frac{\Lambda^{2}}{\mu^{2}}\right)\right. \\
& \left.\left.+\beta_{0}^{2} \log ^{3}\left(\frac{\Lambda^{2}}{\mu^{2}}\right)\right)\right)
\end{aligned}
$$

$C_{1}^{(S)}$ has been computed in the $\overline{M S}$ scheme, in the momentum representation to two loops in [11] and to three loops in [12]. We perform in appendix B.4 its Fourier transform in the coordinate representation:

$$
\begin{aligned}
C_{1}^{(S)}(x)= & \frac{4 \beta_{0}}{\pi^{2} x^{4}} g^{2}(\mu)\left(1+g^{2}(\mu)\left(A_{1,1}^{(S)}+2 \beta_{0} \log \left(x^{2} \mu^{2}\right)\right)+\right. \\
& \left.+g^{4}(\mu)\left(A_{1,2}^{(S)}+A_{1,3}^{(S)} \log \left(x^{2} \mu^{2}\right)+3 \beta_{0}^{2} \log ^{2}\left(x^{2} \mu^{2}\right)\right)\right) \\
+ & \delta^{(4)}(x)\left(4+g^{2}(\mu) A_{1,4}^{(S)}+g^{4}(\mu)\left(A_{1,5}^{(S)}+4 \beta_{1} \log \left(\frac{\Lambda^{2}}{\mu^{2}}\right)\right)\right. \\
& \left.+g^{6}(\mu)\left(A_{1,6}^{(S)}+8 \beta_{2} \log \left(\frac{\Lambda^{2}}{\mu^{2}}\right)-4 \beta_{0} \beta_{1} \log ^{2}\left(\frac{\Lambda^{2}}{\mu^{2}}\right)\right)\right)
\end{aligned}
$$

where:

$$
\begin{aligned}
& \beta_{0}=\frac{1}{(4 \pi)^{2}}\left(\frac{11}{3}-\frac{2}{3} \frac{N_{f}}{N}\right) \\
& \beta_{1}=\frac{1}{(4 \pi)^{4}}\left(\frac{34}{3}-\frac{13}{3} \frac{N_{f}}{N}+\frac{N_{f}}{N^{3}}\right) \\
& \beta_{2}=\frac{1}{(4 \pi)^{6}}\left(\frac{2857}{54}-\frac{1709}{54} \frac{N_{f}}{N}+\frac{56}{27} \frac{N_{f}^{2}}{N^{2}}+\frac{187}{36} \frac{N_{f}}{N^{3}}-\frac{11}{18} \frac{N_{f}^{2}}{N^{4}}+\frac{N_{f}}{4 N^{5}}\right)
\end{aligned}
$$

are the first-three coefficients of the QCD beta function, $\frac{\partial g}{\partial \log \Lambda}=\beta(g)=-\beta_{0} g^{3}-\beta_{1} g^{5}-$ $\beta_{2} g^{7}+\cdots$, and $A_{0, j}^{(S)}, A_{1, j}^{(S)}$ are finite coefficients computed in [11, 12].

$\delta^{(4)}(x)$ and $\Delta^{2} \delta^{(4)}(x)$ are contact terms in the coordinate representation, i.e., distributions supported at coinciding points. They arise from polynomials in the momentum representation. Interestingly, both finite and divergent contact terms occur in the perturbative OPE $[11,13]$. The divergent contact terms require further additive renormalizations [13] with respect to the multiplicative renormalization of $F^{2}$ due to its anomalous dimension. 


\subsection{Verifying eq. (1.3) for $\left\langle F^{2}(z) F^{2}(0)\right\rangle$}

By exploiting the OPE in eq. (3.4), eq. (1.3) reads perturbatively:

$$
4 C_{0}^{(S)}(z)+2 g^{2} \frac{\partial C_{0}^{(S)}(z)}{\partial g^{2}} \sim C_{0}^{(S)}(z) \int C_{1}^{(S)}(x) d^{4} x
$$

where the symbol, $\sim$, means — for the perturbative correlators in the present paper equality of the universal divergent parts.

We test eq. (3.10) in perturbation theory by extracting the divergent parts in both sides from the bare OPE to the order of $g^{2}$ in subsection 4.1 and to the order of $g^{4}$ in subsection 4.2. Indeed, the low-energy theorem is derived for the bare coupling in eqs. (1.3) and (2.2). The perturbative bare coefficients are obtained from the renormalized ones by setting $\mu=\Lambda$ in eqs. (3.5) and (3.6).

Moreover, we observe (subsection 4.1) that we can also match the $z$ dependence of the finite part in the l.h.s. of eq. (3.10), provided that we suitably restrict the IR of the integral in the r.h.s. to the domain $\mathcal{D}_{\frac{1}{z}}^{\Lambda}=\left\{x^{2}: \frac{1}{\Lambda^{2}} \leq x^{2} \leq z^{2}\right\}$.

\subsection{Verifying eq. (1.4) by the RG-improved OPE}

We apply the very same IR subtraction prescription to compute the nonperturbative UV asymptotics in both sides of eq. (5.3), which is the analog version of eq. (3.10) that arises from eqs. (1.4) and (2.9):

$$
\begin{aligned}
& \left(\frac{\beta(g)}{g}\right)^{2} 2 \Lambda_{\mathrm{QCD}}^{2} \frac{\partial C_{0}^{(S)}(z)}{\partial \Lambda_{\mathrm{QCD}}^{2}} \\
& \quad \sim-\left(\frac{\beta(g)}{g}\right)^{2} C_{0}^{(S)}(z) \int_{\mathcal{D}_{\frac{1}{z}}^{\Lambda}} \frac{\beta(g)}{g} C_{1}^{(S)^{\prime}}(x) d^{4} x
\end{aligned}
$$

where the symbol, $\sim$, means - for the RG-improved correlators in the present paper asymptotic equality in the UV as $z \rightarrow 0$.

Eq. (3.11) follows from analog arguments of the perturbative case (subsection 3.1), by taking into account that the leading contribution from the RG-improved OPE is the one that involves the operator $O_{1}$ itself.

Naively, this occurs because the RG-improved version of the OPE is just the resummation of perturbation theory.

In fact, the extra factors of $g^{2}$ that suppress the contributions of other operators in perturbation theory (subsection 3.1) get transformed into extra factors of the running coupling in the RG-improved OPE. Moreover, the IR subtraction prescription that we employ in the r.h.s. effectively amounts to include only contributions in the OPE that arise as all the coordinates in the r.h.s. are uniformly rescaled. In this situation the above argument implies that the leading contribution in the r.h.s. of eq. (1.4) due to the RGimproved OPE only involves the operator $O_{1}$. 
Our computation in section 5 involves the nonperturbative UV asymptotics of the OPE coefficients of $F^{2}$ in the coordinate representation, which we establish a priori, up to a constant overall normalization, by means of the Callan-Symanzik (CS) equation in appendix A, following standard methods worked out in $[15,16]$. Then, we employ the perturbative results in appendix B to fix the overall normalization as well.

Alternatively, following [15] we verify in appendix B.3 and B.6 the nonperturbative UV asymptotics of the RG-invariant coefficients, $\left(\frac{\beta(g)}{g}\right)^{2} C_{0}^{(S)}$ and $-\frac{\beta(g)}{g} C_{1}^{(S)}$, previously derived a priori in appendix A, by means of a change of the perturbative renormalization scheme that allows us to rewrite the perturbative results, originally expressed in terms of $g(\mu)$ and large logs [11-13], in terms of the running coupling, $g(x)$, to the given perturbative order.

\section{Low-energy theorem for $\left\langle F^{2}(z) F^{2}(0)\right\rangle$ in perturbation theory}

Perturbatively, for $n=2$ and $O_{k}=F^{2}$, eq. (1.3) becomes:

$$
4\left\langle F^{2}(z) F^{2}(0)\right\rangle+2 g^{2} \frac{\partial\left\langle F^{2}(z) F^{2}(0)\right\rangle}{\partial g^{2}}=\frac{1}{2} \int\left\langle F^{2}(z) F^{2}(0) F^{2}(x)\right\rangle d^{4} x
$$

since the condensate, $\left\langle F^{2}\right\rangle$, vanishes identically in dimensional regularization to every order in perturbation theory.

We verify eq. (4.1) in perturbation theory for the bare operator $F^{2}$. We choose $z \neq 0$ in order to skip the inessential contact terms in the l.h.s. of eq. (4.1). The 1.h.s. is log divergent [3] to the order of $g^{2}$ because of the nonvanishing anomalous-dimension coefficient, $\gamma_{0}^{\left(F^{2}\right)}=2 \beta_{0}$, of $F^{2}$, where for a canonically normalized operator, $O$ :

$$
\gamma_{O}(g)=-\frac{\partial \log Z^{(O)}}{\partial \log \mu}=-\gamma_{0}^{(O)} g^{2}-\gamma_{1}^{(O)} g^{4}+\cdots
$$

Only $\gamma_{0}^{(O)}$ is scheme independent in general. By a standard argument reported in [15] the anomalous dimension of $F^{2}[22]$ is related to the beta function in $\overline{M S}$-like schemes:

$$
\gamma_{F^{2}}(g)=g \frac{\partial}{\partial g}\left(\frac{\beta(g)}{g}\right)
$$

since the trace of the stress-energy tensor in massless QCD-like theories is [22] RG invariant and proportional to $\frac{\beta(g)}{g} \operatorname{Tr} F^{2}$ in $\overline{M S}$-like schemes. It follows:

$$
\gamma_{F^{2}}(g)=-2 \beta_{0} g^{2}-4 \beta_{1} g^{4}+\cdots
$$

We exhibit in appendix B.3 the scheme where manifestly $\gamma_{1}^{\left(F^{2}\right)}=4 \beta_{1}$ following [15]. It turns out to be the scheme where the constant finite parts of $g^{4} C_{0}^{(S)}(z)$ for $z \neq 0$ vanish to the order of $g^{8}$.

Hence, also the r.h.s. in eq. (4.1) must be divergent, and the divergence can be evaluated by means of the OPE in eq. (3.4) [3]. Thus, it suffices to evaluate perturbatively the 3-point correlator, $\left\langle F^{2}(z) F^{2}(0) F^{2}(x)\right\rangle$, by fixing $z$ while $x$ may be close either to $z$ or 0 . 
For $x$ close to 0 we get:

$$
\left\langle F^{2}(z) F^{2}(0) F^{2}(x)\right\rangle=C_{1}^{(S)}(x)\left\langle F^{2}(z) F^{2}(0)\right\rangle+\ldots
$$

Therefore, as far as the divergent parts are concerned, the low-energy theorem reads (subsection 3.3):

$$
4 C_{0}^{(S)}(z)+2 g^{2} \frac{\partial C_{0}^{(S)}(z)}{\partial g^{2}} \sim C_{0}^{(S)}(z) \int C_{1}^{(S)}(x) d^{4} x
$$

where a factor of 2 has been included in the r.h.s. to take into account that $x$ can be close either to $z$ or 0 [3].

To evaluate eq. (4.6) we employ in the r.h.s. the perturbative version of $C_{1}^{(S)}$. After extracting from $C_{1}^{(S)}$ the lowest-order contact term:

$$
C_{1}^{(S)}(x)=4 \delta^{4}(x)+C_{1}^{(S)^{\prime}}(x)
$$

Eq. (4.6) simplifies significantly:

$$
2 g^{2} \frac{\partial C_{0}^{(S)}(z)}{\partial g^{2}} \sim C_{0}^{(S)}(z) \int C_{1}^{(S)^{\prime}}(x) d^{4} x
$$

Interestingly, the lowest-order contact term is crucial to satisfy eq. (4.6) to the order of $g^{0}$. In [3] both this contact term and the compensating first term in the l.h.s. of eq. (4.6) have been skipped (subsection 4.1).

\subsection{Order of $g^{2}$}

To verify eq. (4.8) to the order of $g^{2}[3]$, we employ the corresponding bare OPE coefficients in the coordinate representation. They are obtained simply setting $\mu=\Lambda$ in the renormalized ones:

$$
\begin{aligned}
C_{0}^{(S)}(z) & =\frac{N^{2}-1}{z^{8}} \frac{48}{\pi^{4}}\left(1+g^{2}(\Lambda)\left(A_{0,1}^{(S)}+2 \beta_{0} \log \left(\frac{\Lambda^{2}}{\mu^{2}}\right)+2 \beta_{0} \log \left(z^{2} \mu^{2}\right)\right)\right) \\
C_{1}^{(S)^{\prime}}(x) & =\frac{1}{x^{4}} \frac{4 \beta_{0}}{\pi^{2}} g^{2}(\Lambda)
\end{aligned}
$$

In eq. (4.9) we have skipped the inessential contact terms in $C_{0}^{(S)}(z)$ by choosing $z \neq 0$. Hence, the divergent part of eq. (4.8) reads to the order of $g^{2}$ :

$$
\frac{N^{2}-1}{z^{8}} \frac{48}{\pi^{4}} g^{2}(\Lambda) 4 \beta_{0} \log \left(\frac{\Lambda^{2}}{\mu^{2}}\right)=\frac{N^{2}-1}{z^{8}} \frac{48}{\pi^{4}} \frac{4 \beta_{0}}{\pi^{2}} g^{2}(\Lambda) \int \frac{1}{x^{4}} d^{4} x
$$

The integral in the r.h.s. is both UV and IR divergent [3].

Incidentally, this divergence plays a key role for the compatibility of the open/closed string duality with perturbation theory in massless QCD [3]. 
We regularize the integral by restricting to the domain $\mathcal{D}_{\mu}^{\Lambda}=\left\{x^{2}: \frac{1}{\Lambda^{2}} \leq x^{2} \leq \frac{1}{\mu^{2}}\right\}$. Hence, performing the integral in polar coordinates, we get:

$$
\int_{\mathcal{D}_{\mu}^{\Lambda}} \frac{1}{x^{4}} d^{4} x=\pi^{2} \log \left(\frac{\Lambda^{2}}{\mu^{2}}\right)
$$

that implies eq. (4.11) according to [3].

Our key observation is that we can also match the $z$ dependence of the finite part in the l.h.s. of eq. (4.8), provided that we suitably modify the integration domain in the IR, $\mathcal{D}_{\frac{1}{z}}^{\Lambda}=\left\{x^{2}: \frac{1}{\Lambda^{2}} \leq x^{2} \leq z^{2}\right\}$. By including the constant finite parts for future employment, we get to the order of $g^{2}$ :

$$
\begin{aligned}
& \frac{N^{2}-1}{z^{8}} \frac{48}{\pi^{4}} g^{2}(\Lambda)\left(2 A_{0,1}^{(S)}+4 \beta_{0} \log \left(z^{2} \Lambda^{2}\right)\right) \\
& \quad=\frac{N^{2}-1}{z^{8}} \frac{48}{\pi^{4}}\left(A_{1,4}^{(S)}+\frac{4 \beta_{0}}{\pi^{2}} \int_{\mathcal{D}_{\frac{1}{z}}^{\Lambda}} \frac{1}{x^{4}} d^{4} x\right) g^{2}(\Lambda)
\end{aligned}
$$

where now the equality also includes the $z$ dependence up to the constant finite parts. This prescription plays a key role for getting the correct nonperturbative RG-improved UV asymptotics in the r.h.s. of eq. (3.11).

Of course, any prescription for the IR cutoff in the r.h.s. leads, already to the order of $g^{2}$, to an ambiguity for the constant finite parts in the r.h.s.

Presently, we cannot resolve this finite ambiguity in the framework of our computation that is either based in this section on the perturbative OPE or in section 5 on its universal nonperturbative asymptotics.

\subsection{Order of $g^{4}$}

The $O\left(g^{2}\right)$ UV log divergence computed in subsection 4.1 is universal, i.e., it depends only on the first coefficient of the anomalous dimension. The $O\left(g^{4}\right) \log$-squared divergence in the l.h.s. of eq. (4.8) is universal as well, because it is essentially the square of the $O\left(g^{2}\right)$ log divergence.

Instead, the $O\left(g^{4}\right) \log$ divergence is scheme dependent, and therefore depends on the constant finite parts to the order of $g^{2}$. Thus, we verify perturbatively eq. (4.8) by limiting ourselves to the universal divergences.

However, we compute as well the scheme-dependent finite and log-divergent parts in both sides of eq. (4.8) to the order of $g^{4}$ for future employment. We evaluate the r.h.s. of eq. (4.8) to the order of $g^{4}$ :

$$
\begin{aligned}
& \frac{N^{2}-1}{z^{8}} \frac{48}{\pi^{4}}\left(1+g^{2}(\Lambda)\left(A_{0,1}^{(S)}+2 \beta_{0} \log \left(z^{2} \Lambda^{2}\right)\right)\right) \\
& g^{2}(\Lambda) \int_{\mathcal{D}_{\frac{1}{z}}^{\Lambda}} \frac{4 \beta_{0}}{\pi^{2} x^{4}}\left(1+g^{2}(\Lambda)\left(A_{1,1}^{(S)}+2 \beta_{0} \log \left(x^{2} \Lambda^{2}\right)\right)\right) \\
& +\delta^{(4)}(x)\left(A_{1,4}^{(S)}+g^{2}(\Lambda)\left(A_{1,5}^{(S)}+4 \beta_{1} \log \left(\frac{\Lambda^{2}}{\mu^{2}}\right)\right)\right) d^{4} x
\end{aligned}
$$


It reads to the order of $g^{4}$ :

$$
\begin{aligned}
& \frac{N^{2}-1}{z^{8}} \frac{48}{\pi^{4}} g^{4}(\Lambda)\left(\int_{\mathcal{D}_{\frac{1}{z}}^{\Lambda}} \frac{4 \beta_{0}}{\pi^{2} x^{4}}\left(A_{1,1}^{(S)}+2 \beta_{0} \log \left(x^{2} \Lambda^{2}\right)\right) d^{4} x+A_{1,5}^{(S)}\right. \\
& \left.+4 \beta_{1} \log \left(\frac{\Lambda^{2}}{\mu^{2}}\right)\right)+\frac{N^{2}-1}{z^{8}} \frac{48}{\pi^{4}} g^{4}(\Lambda)\left(A_{0,1}^{(S)}+2 \beta_{0} \log \left(z^{2} \Lambda^{2}\right)\right) \int_{\mathcal{D}_{\frac{1}{z}}^{\Lambda}} \frac{4 \beta_{0}}{\pi^{2} x^{4}} d^{4} x \\
& +\frac{N^{2}-1}{z^{8}} \frac{48}{\pi^{4}} g^{4}(\Lambda)\left(A_{0,1}^{(S)}+2 \beta_{0} \log \left(z^{2} \Lambda^{2}\right)\right) A_{1,4}^{(S)}
\end{aligned}
$$

Hence, we evaluate eq. (4.8) to the order of $g^{4}$ :

$$
\begin{aligned}
& \frac{N^{2}-1}{z^{8}} \frac{48}{\pi^{4}} g^{4}(\Lambda)\left(4 A_{0,2}^{(S)}+4 A_{0,3}^{(S)} \log \left(z^{2} \Lambda^{2}\right)+12 \beta_{0}^{2} \log ^{2}\left(z^{2} \Lambda^{2}\right)\right) \\
& \sim \frac{N^{2}-1}{z^{8}} \frac{48}{\pi^{4}} g^{4}(\Lambda)\left(\int_{\mathcal{D}_{\frac{1}{z}}^{\Lambda}} \frac{4 \beta_{0}}{\pi^{2} x^{4}}\left(A_{1,1}^{(S)}+2 \beta_{0} \log \left(x^{2} \Lambda^{2}\right)\right) d^{4} x+A_{1,5}^{(S)}\right. \\
& \left.\quad+4 \beta_{1} \log \left(\frac{\Lambda^{2}}{\mu^{2}}\right)\right)+\frac{N^{2}-1}{z^{8}} \frac{48}{\pi^{4}} g^{4}(\Lambda)\left(A_{0,1}^{(S)}+2 \beta_{0} \log \left(z^{2} \Lambda^{2}\right)\right) \int_{\mathcal{D}_{\frac{1}{z}}^{\Lambda}} \frac{4 \beta_{0}}{\pi^{2} x^{4}} d^{4} x \\
& \quad+\frac{N^{2}-1}{z^{8}} \frac{48}{\pi^{4}} g^{4}(\Lambda)\left(A_{0,1}^{(S)}+2 \beta_{0} \log \left(z^{2} \Lambda^{2}\right)\right) A_{1,4}^{(S)} \\
& =\frac{N^{2}-1}{z^{8}} \frac{48}{\pi^{4}} g^{4}(\Lambda)\left(4 \beta_{0} A_{1,1}^{(S)} \log \left(z^{2} \Lambda^{2}\right)+4 \beta_{0}^{2} \log ^{2}\left(z^{2} \Lambda^{2}\right)+A_{1,5}^{(S)}\right. \\
& \left.\quad+4 \beta_{1} \log \left(\frac{\Lambda^{2}}{\mu^{2}}\right)\right)+\frac{N^{2}-1}{z^{8}} \frac{48}{\pi^{4}} g^{4}(\Lambda)\left(A_{0,1}^{(S)}+2 \beta_{0} \log \left(z^{2} \Lambda^{2}\right)\right) 4 \beta_{0} \log \left(z^{2} \Lambda^{2}\right) \\
& \quad+\frac{N^{2}-1}{z^{8}} \frac{48}{\pi^{4}} g^{4}(\Lambda)\left(A_{0,1}^{(S)}+2 \beta_{0} \log \left(z^{2} \Lambda^{2}\right)\right) A_{1,4}^{(S)} \\
& =\frac{N^{2}-1}{z^{8}} \frac{48}{\pi^{4}} g^{4}(\Lambda)\left(4 \beta_{0} A_{1,1}^{(S)} \log \left(z^{2} \Lambda^{2}\right)+4 \beta_{0}^{2} \log ^{2}\left(z^{2} \Lambda^{2}\right)+A_{1,5}^{(S)}\right. \\
& \left.\quad+4 \beta_{1} \log \left(\frac{\Lambda^{2}}{\mu^{2}}\right)\right)+\frac{N^{2}-1}{z^{8}} \frac{48}{\pi^{4}} g^{4}(\Lambda)\left(A_{0,1}^{(S)} A_{1,4}^{(S)}+8 \beta_{0}^{2} \log ^{2}\left(z^{2} \Lambda^{2}\right)\right. \\
& \left.\quad+2 \beta_{0} A_{1,4}^{(S)} \log \left(z^{2} \Lambda^{2}\right)+4 \beta_{0} A_{0,1}^{(S)} \log \left(z^{2} \Lambda^{2}\right)\right) \\
& =\frac{N^{2}-1}{z^{8}} \frac{48}{\pi^{4}} g^{4}(\Lambda)\left(A_{1,5}^{(S)}+A_{0,1}^{(S)} A_{1,4}^{(S)}+4 \beta_{0}\left(A_{1,1}^{(S)}+A_{0,1}^{(S)}+\frac{1}{2} A_{1,4}^{(S)}\right) \log \left(z^{2} \Lambda^{2}\right)\right. \\
& \left.+4 \beta_{1} \log \left(\frac{\Lambda^{2}}{\mu^{2}}\right)+12 \beta_{0}^{2} \log { }^{2}\left(z^{2} \Lambda^{2}\right)\right)
\end{aligned}
$$

where we recall that the symbol, $\sim$, means - for the perturbative correlators in the present paper - equality of the universal divergent parts.

Therefore, the universal log-squared divergences in both sides of eq. (4.8) agree. 


\section{Nonperturbative UV asymptotics of the low-energy theorem for $\left\langle F^{2}(z) F^{2}(0)\right\rangle$}

We compute now the nonperturbative UV asymptotics of the low-energy theorem for $\left\langle F^{2}(z) F^{2}(0)\right\rangle$ in massless QCD, within the universal leading and next to leading logarithmic accuracy, by means of the UV asymptotics of the renormalized OPE coefficients in appendix A, and of their perturbative normalization in appendix B.

It is convenient to introduce the RG-invariant coefficients $\left(\frac{\beta(g)}{g}\right)^{2} C_{0}^{(S)}(z)$ and $-\frac{\beta(g)}{g} C_{1}^{(S)}(x)$ associated to the OPE of the RG-invariant operator $-\frac{\beta(g)}{g} F^{2}$.

The change of normalization does not affect the universal UV asymptotics but for the overall normalization, yet it is specifically convenient for the perturbative computations in appendix B: the universal UV asymptotics of the 2-point correlators of $-\frac{\beta(g)}{g} F^{2}$ that is RG invariant, of $F^{2}$ that has a nontrivial anomalous dimension, and of $g^{2} F^{2}$ whose first coefficient of the anomalous dimension vanishes (appendix B.3), coincide up to the overall normalization [15].

The UV universal asymptotics of the RG-invariant OPE coefficients reads:

$$
\begin{aligned}
\left(\frac{\beta(g)}{g}\right)^{2} C_{0}^{(S)}(z) & \sim \frac{N^{2}-1}{z^{8}} \frac{48}{\pi^{4}} \beta_{0}^{2} g^{4}(z) \\
& \sim \frac{N^{2}-1}{\pi^{4}} \frac{48}{z^{8}} \frac{1}{\log ^{2}\left(\frac{1}{z^{2} \Lambda_{\mathrm{QCD}}^{2}}\right)}\left(1-2 \frac{\beta_{1}}{\beta_{0}^{2}} \frac{\log \log \left(\frac{1}{z^{2} \Lambda_{\mathrm{QCD}}^{2}}\right)}{\log \left(\frac{1}{z^{2} \Lambda_{\mathrm{QCD}}^{2}}\right)}\right)
\end{aligned}
$$

and:

$$
\begin{aligned}
-\frac{\beta(g)}{g} C_{1}^{(S)^{\prime}}(x) & \sim \frac{4 \beta_{0}^{2}}{\pi^{2}} \frac{1}{x^{4}} g^{4}(x) \\
& \sim \frac{4}{\pi^{2}} \frac{1}{x^{4}} \frac{1}{\log ^{2}\left(\frac{1}{x^{2} \Lambda_{\mathrm{QCD}}^{2}}\right)}\left(1-2 \frac{\beta_{1}}{\beta_{0}^{2}} \frac{\log \log \left(\frac{1}{x^{2} \Lambda_{\mathrm{QCD}}^{2}}\right)}{\log \left(\frac{1}{x^{2} \Lambda_{\mathrm{QCD}}^{2}}\right)}\right)
\end{aligned}
$$

where we recall that the symbol, $\sim$, means - for the RG-improved correlators in the present paper - asymptotic equality in the UV as $z, x \rightarrow 0$. The asymptotic equalities in the second lines of eqs. (5.1) and (5.2) follow from eq. (A.13).

It is convenient to employ the version of the low-energy theorem that involves $\Lambda_{\mathrm{QCD}}$ and the canonical normalization of the YM action in eq. (1.4). We skip the finite contact term in $C_{1}^{(S)}$, the compensating term in the 1.h.s. of eq. (1.4), and the divergent contact terms in $C_{1}^{(S)}$ that, according to [13], should be renormalized to zero.

Then, for the renormalized correlators, it should hold nonperturbatively and asymptotically as $z \rightarrow 0$ (subsection 3.4 ):

$$
\begin{aligned}
& \left(\frac{\beta(g)}{g}\right)^{2} 2 \Lambda_{\mathrm{QCD}}^{2} \frac{\partial C_{0}^{(S)}(z)}{\partial \Lambda_{\mathrm{QCD}}^{2}} \\
& \quad \sim-\left(\frac{\beta(g)}{g}\right)^{2} C_{0}^{(S)}(z) \int_{\mathcal{D}_{\frac{1}{z}}^{\Lambda}} \frac{\beta(g)}{g} C_{1}^{(S)^{\prime}}(x) d^{4} x
\end{aligned}
$$


Firstly, we compute the 1.h.s. of eq. (5.3):

$$
\begin{aligned}
& \left(\frac{\beta(g)}{g}\right)^{2} 2 \Lambda_{\mathrm{QCD}}^{2} \frac{\partial C_{0}^{(S)}(z)}{\partial \Lambda_{\mathrm{QCD}}^{2}} \\
& \sim \frac{N^{2}-1}{\pi^{4}} \frac{48}{z^{8}} \frac{4}{\log ^{3}\left(\frac{1}{z^{2} \Lambda_{\mathrm{QCD}}^{2}}\right)}\left(1-3 \frac{\beta_{1}}{\beta_{0}^{2}} \frac{\log \log \left(\frac{1}{z^{2} \Lambda_{\mathrm{QCD}}^{2}}\right)}{\log \left(\frac{1}{z^{2} \Lambda_{\mathrm{QCD}}^{2}}\right)}\right) \\
& \sim \frac{N^{2}-1}{\pi^{4}} \frac{48}{z^{8}} 4 \beta_{0}^{3} g^{6}(z)
\end{aligned}
$$

In the r.h.s. of eq. (5.3) the crucial step is the integration of $-\frac{\beta(g)}{g} C_{1}^{(S)}$. According to the prescription in subsection 4.1 , we have restricted the integral to the domain $\mathcal{D}_{\frac{1}{z}}^{\Lambda}=\left\{x^{2}\right.$ : $\left.\frac{1}{\Lambda^{2}} \leq x^{2} \leq z^{2}\right\}$.

But now, after the RG resummation, the integral is UV convergent because of the asymptotic freedom, and therefore we can remove the UV cutoff. Thus, we may extend the integration to the new domain $\mathcal{D}_{\frac{1}{z}}^{\infty}=\left\{x^{2}: 0 \leq x^{2} \leq z^{2}\right\}$.

Incidentally, the nonperturbative UV finiteness of the integral in eq. (5.3), as opposed to the UV divergence of the integral in eq. (4.11) in perturbation theory, plays a key role for the no-go theorem in [3]. We get:

$$
\begin{aligned}
& -\int_{\mathcal{D}_{\frac{1}{z}}^{\infty}} \frac{\beta(g)}{g} C_{1}^{(S)^{\prime}}(x) d^{4} x \\
& \sim \int_{\mathcal{D}_{\frac{1}{z}}^{\infty}} \frac{4}{\pi^{2} x^{4}} \frac{1}{\log ^{2}\left(\frac{1}{x^{2} \Lambda_{\mathrm{QCD}}^{2}}\right)}\left(1-2 \frac{\beta_{1}}{\beta_{0}^{2}} \frac{\log \log \left(\frac{1}{x^{2} \Lambda_{\mathrm{QCD}}^{2}}\right)}{\log \left(\frac{1}{x^{2} \Lambda_{\mathrm{QCD}}^{2}}\right)}\right) d^{4} x \\
& \quad \sim 4 \frac{1}{\log \left(\frac{1}{z^{2} \Lambda_{\mathrm{QCD}}^{2}}\right)}\left(1-\frac{\beta_{1}}{\beta_{0}^{2}} \frac{\log \log \left(\frac{1}{z^{2} \Lambda_{\mathrm{QCD}}^{2}}\right)}{\log \left(\frac{1}{z^{2} \Lambda_{\mathrm{QCD}}^{2}}\right)}\right) \\
& \sim 4 \beta_{0} g^{2}(z)
\end{aligned}
$$

that substituted in the r.h.s. of eq. (3.11) implies:

$$
\begin{aligned}
& -\left(\frac{\beta(g)}{g}\right)^{2} C_{0}^{(S)}(z) \int_{\mathcal{D}_{\frac{1}{z}}^{\infty}} \frac{\beta(g)}{g} C_{1}^{(S)^{\prime}}(x) d^{4} x \\
& \sim \frac{N^{2}-1}{z^{8}} \frac{48}{\pi^{4}} \beta_{0}^{2} g^{4}(z) 4 \beta_{0} g^{2}(z) \\
& \sim \frac{N^{2}-1}{z^{8}} \frac{48}{\pi^{4}} 4 \beta_{0}^{3} g^{6}(z)
\end{aligned}
$$

that actually matches eq. (5.4). 
Just as an aside, the integral in eq. (5.5) is computed in polar coordinates by means of the obvious change of variables and by integrating by parts:

$$
\begin{aligned}
& \int_{\mathcal{D}_{\frac{1}{z}}^{\infty}} \frac{1}{\pi^{2} x^{4}} \frac{1}{\log ^{2}\left(\frac{1}{x^{2} \Lambda_{\mathrm{QCD}}^{2}}\right)}\left(1-2 \frac{\beta_{1}}{\beta_{0}^{2}} \frac{\log \log \left(\frac{1}{x^{2} \Lambda_{\mathrm{QCD}}^{2}}\right)}{\log \left(\frac{1}{x^{2} \Lambda_{\mathrm{QCD}}^{2}}\right)}\right) d^{4} x \\
& =\int_{0}^{|z|} \frac{2}{\log ^{2}\left(\frac{1}{|x|^{2} \Lambda_{\mathrm{QCD}}^{2}}\right)}\left(1-2 \frac{\beta_{1}}{\beta_{0}^{2}} \frac{\log \log \left(\frac{1}{|x|^{2} \Lambda_{\mathrm{QCD}}^{2}}\right)}{\log \left(\frac{1}{|x|^{2} \Lambda_{\mathrm{QCD}}^{2}}\right)}\right) \frac{d|x|}{|x|} \\
& =\frac{1}{\log \frac{1}{z^{2} \Lambda_{\mathrm{QCD}}^{2}}}-\frac{\beta_{1}}{\beta_{0}^{2}} \frac{\log \log \frac{1}{z^{2} \Lambda_{\mathrm{QCD}}^{2}}}{\log ^{2} \frac{\beta_{1}}{z^{2} \Lambda_{\mathrm{QCD}}^{2}}} \frac{1}{2 \beta_{0}^{2}} \frac{1}{\log ^{2} \frac{1}{z^{2} \Lambda_{\mathrm{QCD}}^{2}}} \\
& \sim \frac{1}{\log \left(\frac{1}{z^{2} \Lambda_{\mathrm{QCD}}^{2}}\right)}\left(1-\frac{\beta_{1}}{\beta_{0}^{2}} \frac{\log \log \left(\frac{1}{z^{2} \Lambda_{\mathrm{QCD}}^{2}}\right)}{\log \left(\frac{1}{z^{2} \Lambda_{\mathrm{QCD}}^{2}}\right)}\right)
\end{aligned}
$$

with $|x|=\sqrt{x^{2}}$.

\section{Perturbative OPE to the order of $g^{2}$ and nonperturbative UV asymptotics from the low-energy theorem for $\langle O(z) O(0)\rangle$}

By inverting the arguments in the preceding sections, we now employ the low-energy theorem in order to get information on the OPE coefficients.

We consider, in a perturbatively massless QCD-like theory, the operators, $O_{i}$, mentioned in subsection 3.1 that we assume to be gauge invariant, to have the same canonical dimension, and to mix under renormalization. Then, for $n=2$ eq. (2.8) reads:

$$
\begin{aligned}
& 2\left(c_{i}+c_{k}\right)\left\langle O_{i}(z) O_{k}(0)\right\rangle+2 \frac{\partial\left\langle O_{i}(z) O_{k}(0)\right\rangle}{\partial \log g} \\
& \quad=\int\left\langle O_{i}(z) O_{k}(0) F^{2}(x)\right\rangle-\left\langle O_{i}(z) O_{k}(0)\right\rangle\left\langle F^{2}(x)\right\rangle d^{4} x
\end{aligned}
$$

By exploiting the OPE in eq. (3.3), we get perturbatively:

$$
\begin{aligned}
& 2\left(c_{i}+c_{k}\right)\left\langle O_{i}(z) O_{k}(0)\right\rangle+2 \frac{\partial\left\langle O_{i}(z) O_{k}(0)\right\rangle}{\partial \log g} \\
& \sim\left\langle O_{i}(z) O_{k}(0)\right\rangle \int C_{O_{i}}^{\left(F^{2}, O_{i}\right)}(x-z)+C_{O_{k}}^{\left(F^{2}, O_{k}\right)}(x) d^{4} x \\
& \quad+\sum_{l \neq i, k}\left\langle O_{l}(z) O_{k}(0)\right\rangle \int C_{O_{l}}^{\left(F^{2}, O_{i}\right)}(x-z) d^{4} x \\
& \quad+\sum_{l \neq k, i}\left\langle O_{i}(z) O_{l}(0)\right\rangle \int C_{O_{l}}^{\left(F^{2}, O_{k}\right)}(x) d^{4} x
\end{aligned}
$$

As the operators $O_{i}$ are canonically normalized, only the first term in the l.h.s. of eq. (6.2) contributes perturbatively to the order of $g^{0}$. Moreover, to this order, we get:

$$
2 c_{i}\left\langle O_{i}(z) O_{i}(0)\right\rangle=\left\langle O_{i}(z) O_{i}(0)\right\rangle \int C_{O_{i}}^{\left(F^{2}, O_{i}\right)}(x) d^{4} x
$$


since, because of the orthogonality of the operators with themselves and with the operators with different canonical dimensions, all the remaining contributions in the r.h.s. from the complete OPE vanish to this order.

Hence, as the l.h.s. is finite, a finite contact term must occur, for $c_{i} \neq 0$, to the order of $g^{0}$. Indeed, by dimensional analysis, since $F^{2}$ has canonical dimension 4 :

$$
C_{O_{i}}^{\left(F^{2}, O_{i}\right)}(x)=2 c_{i} \delta^{4}(x)+C_{O_{i}}^{\left(F^{2}, O_{i}\right)^{\prime}}(x)
$$

with $C_{O_{i}}^{\left(O_{i}, F^{2}\right)^{\prime}}$ necessarily on the order of $g^{2}$ - for nontrivial anomalous dimensions of the operators $O_{i}$ - because, by dimensional analysis and the orthogonality of the operators to the order of $g^{2}$, it has to produce in the r.h.s. the log divergence that matches the one in the lhs, which may only arise to the order of $g^{2}$ because of the log derivative in the lhs:

$$
\frac{\partial\left\langle O_{i}(z) O_{i}(0)\right\rangle}{\partial \log g} \sim\left\langle O_{i}(z) O_{i}(0)\right\rangle \int C_{O_{i}}^{\left(F^{2}, O_{i}\right)^{\prime}}(x) d^{4} x
$$

Thus, we have reduced our computation to the case of a single multiplicatively renomalizable operator with well-defined anomalous dimension, which we summarize as follows.

The low-energy theorem for any 2-point correlator, $\langle O(z) O(0)\rangle$, of a canonically normalized gauge-invariant operator, $O$, belonging to the aforementioned orthogonal basis, implies perturbatively:

$$
2 c C_{0}^{(O)}(z)+\frac{\partial C_{0}^{(O)}(z)}{\partial \log g} \sim C_{0}^{(O)}(z) \int C_{O}^{\left(F^{2}, O\right)}(x) d^{4} x
$$

with $c$ the exponent, defined in subsection 2.3, of the canonical rescaling of the operator $O$, and the OPE coefficients defined in eqs. (A.1) and (A.2). For an operator of spin $s$, the bare $C_{0}^{(O)}$, up to a finite term on the order of $g^{2}$, reads to the order of $g^{2}$ for $z \neq 0$ :

$$
C_{0}^{(O)}(z)=A^{(s)} \frac{P^{(s)}(z)}{z^{2 D}}\left(1+g^{2}(\Lambda) \gamma_{0}^{(O)} \log \left(z^{2} \Lambda^{2}\right)\right)
$$

with $P^{(s)}(z)$ the spin projector in the coordinate representation in the conformal limit, $A^{(s)}$ a constant normalization factor, $D$ the canonical dimension, and $\gamma_{0}^{(O)}$ the first coefficient of the anomalous dimension.

The first term in the 1.h.s. of eq. (6.6) implies that a finite contact term must occur for $c \neq 0$ to the order of $g^{0}$ :

$$
C_{O}^{\left(F^{2}, O\right)}(x)=2 c \delta^{4}(x)+C_{O}^{\left(F^{2}, O\right)^{\prime}}(x)
$$

By skipping the contact term in the r.h.s. and the compensating term in the 1.h.s. of eq. (6.6), eq. (6.5) implies to the order of $g^{2}$ :

$$
\frac{2 A^{(s)} \gamma_{0}^{(O)} P^{(s)}(z)}{z^{2 D}} g^{2}(\Lambda) \log \left(z^{2} \Lambda^{2}\right) \sim \frac{A^{(s)} P^{(s)}(z)}{z^{2 D}} \int_{\mathcal{D}_{\frac{1}{z}}^{\Lambda}} C_{O}^{\left(F^{2}, O\right)^{\prime}}(x) d^{4} x
$$


This fixes $C_{O}^{\left(F^{2}, O\right)}$ in a massless QCD-like theory to the order of $g^{2}$ in terms of the first coefficient of the anomalous dimension, $\gamma_{0}^{(O)}$, and of the exponent, $c$, of the canonical rescaling:

$$
C_{O}^{\left(F^{2}, O\right)}(x)=2 c \delta^{4}(x)+g^{2}(\Lambda) \frac{2 \gamma_{0}^{(O)}}{\pi^{2} x^{4}}
$$

Moreover, it follows from eq. (A.20):

$$
\begin{aligned}
C_{O}^{\left(F^{2}, O\right)^{\prime}}(x) & \sim \frac{2 \gamma_{0}^{(O)}}{\pi^{2} x^{4}} g^{2}(x)\left(\frac{g(x)}{g(\mu)}\right)^{2} \\
& \sim \frac{2 \gamma_{0}^{(O)}}{\pi^{2} x^{4}} \frac{1}{g^{2}(\mu) \beta_{0}^{2} \log ^{2}\left(\frac{1}{x^{2} \Lambda_{\mathrm{QCD}}^{2}}\right)}\left(1-2 \frac{\beta_{1}}{\beta_{0}^{2}} \frac{\log \log \left(\frac{1}{x^{2} \Lambda_{\mathrm{QCD}}^{2}}\right)}{\log \left(\frac{1}{x^{2} \Lambda_{\mathrm{QCD}}^{2}}\right)}\right)
\end{aligned}
$$

Then, the low-energy theorem implies asymptotically as $z \rightarrow 0$ :

$$
2 \Lambda_{\mathrm{QCD}}^{2} \frac{\partial C_{0}^{(O)}(z)}{\partial \Lambda_{\mathrm{QCD}}^{2}} \sim-\frac{\beta(g)}{g} C_{0}^{(O)}(z) \int_{\mathcal{D}_{\frac{1}{z}}^{\Lambda}} C_{O}^{\left(F^{2}, O\right)^{\prime}}(x) d^{4} x
$$

with:

$$
\begin{aligned}
C_{0}^{(O)}(z) & \sim A^{(s)} \frac{P^{(s)}(z)}{z^{2 D}}\left(\frac{g(z)}{g(\mu)}\right)^{\frac{2 \gamma_{0}^{(O)}}{\beta_{0}}} \\
& \sim \frac{1}{z^{2 D}} \frac{A^{(s)} P^{(s)}(z)}{\left(g^{2}(\mu)\right)^{\frac{\gamma_{0}^{(O)}}{\beta_{0}}} \beta_{0}^{\frac{\gamma_{0}^{(O)}}{\beta_{0}}} \log \frac{\gamma_{0}^{(O)}}{\beta_{0}}\left(\frac{1}{z^{2} \Lambda_{\mathrm{QCD}}^{2}}\right)}\left(1-\frac{\gamma_{0}^{(O)}}{\beta_{0}} \frac{\beta_{1}}{\beta_{0}^{2}} \frac{\log \log \left(\frac{1}{z^{2} \Lambda_{\mathrm{QCD}}^{2}}\right)}{\log \left(\frac{1}{z^{2} \Lambda_{\mathrm{QCD}}^{2}}\right)}\right)
\end{aligned}
$$

The l.h.s. of eq. (6.12) reads:

$$
\begin{aligned}
& 2 \Lambda_{\mathrm{QCD}}^{2} \frac{\partial C_{0}^{(O)}(z)}{\partial \Lambda_{\mathrm{QCD}}^{2}} \\
& \sim \frac{2 \gamma_{0}^{(O)}}{z^{2 D}} \frac{A^{(s)} P^{(s)}(z)}{\left(g^{2}(\mu)\right)^{\frac{\gamma_{0}^{(O)}}{\beta_{0}}} \beta_{0}^{1+\frac{\gamma_{0}^{(O)}}{\beta_{0}}} \log ^{1+\frac{\gamma_{0}^{(O)}}{\beta_{0}}}\left(\frac{1}{z^{2} \Lambda_{\mathrm{QCD}}^{2}}\right)}\left(1-\left(1+\frac{\gamma_{0}^{(O)}}{\beta_{0}}\right) \frac{\beta_{1}}{\beta_{0}^{2}} \frac{\log \log \left(\frac{1}{z^{2} \Lambda_{\mathrm{QCD}}^{2}}\right)}{\log \left(\frac{1}{z^{2} \Lambda_{\mathrm{QCD}}^{2}}\right)}\right) \\
& \sim A^{(s)} P^{(s)}(z) \frac{2 \gamma_{0}^{(O)}}{z^{2 D}}\left(\frac{g(z)}{g(\mu)}\right)^{\frac{2 \gamma_{0}^{(O)}}{\beta_{0}}} g^{2}(z)
\end{aligned}
$$

The integral in the r.h.s. of eq. (6.12) is UV convergent exactly as in section 5, and the 
integration domain can be extended to $\mathcal{D}_{\frac{1}{z}}^{\infty}$ :

$$
\begin{aligned}
& -\frac{\beta(g)}{g} \int_{\mathcal{D}_{\frac{1}{z}}^{\infty}} C_{O}^{\left(F^{2}, O\right)^{\prime}}(x) d^{4} x \\
& \sim \int_{\mathcal{D}_{\frac{1}{z}}^{\infty}} \frac{2 \gamma_{0}^{(O)} \beta_{0}}{\pi^{2} x^{4}} \frac{1}{\beta_{0}^{2} \log ^{2}\left(\frac{1}{x^{2} \Lambda_{\mathrm{QCD}}^{2}}\right)}\left(1-2 \frac{\beta_{1}}{\beta_{0}^{2}} \frac{\log \log \left(\frac{1}{x^{2} \Lambda_{\mathrm{QCD}}^{2}}\right)}{\log \left(\frac{1}{x^{2} \Lambda_{\mathrm{QCD}}^{2}}\right)}\right) d^{4} x \\
& \sim \frac{2 \gamma_{0}^{(O)}}{\beta_{0} \log \left(\frac{1}{z^{2} \Lambda_{\mathrm{QCD}}^{2}}\right)}\left(1-\frac{\beta_{1}}{\beta_{0}^{2}} \frac{\log \log \left(\frac{1}{z^{2} \Lambda_{\mathrm{QCD}}^{2}}\right)}{\log \left(\frac{1}{z^{2} \Lambda_{\mathrm{QCD}}^{2}}\right)}\right) \sim 2 \gamma_{0}^{(O)} g^{2}(z)
\end{aligned}
$$

Therefore, the r.h.s. of eq. (6.12) reads:

$$
-\frac{\beta(g)}{g} C_{0}^{(O)}(z) \int_{\mathcal{D}_{\frac{1}{z}}^{\infty}} C_{O}^{\left(F^{2}, O\right)^{\prime}}(x) d^{4} x \sim \frac{A^{(s)} P^{(s)}(z)}{z^{2 D}}\left(\frac{g(z)}{g(\mu)}\right)^{\frac{2 \gamma_{0}^{(O)}}{\beta_{0}}} 2 \gamma_{0}^{(O)} g^{2}(z)
$$

that actually matches eq. (6.14).

\section{Conclusions}

\subsection{Low-energy theorem and perturbation theory}

As expected, our computations have actually verified the equality to the order of $g^{4}$ of the perturbative universal divergent parts in both sides of eq. (1.3), for $n=2$ and $O_{k}=F^{2}$ (section 4), by means of the OPE of the operator $F^{2}$ with itself worked out in [11-13].

The contact terms in $C_{0}^{(S)}$ have been, in fact, inessential to verify the low-energy theorem, since they can be skipped by choosing $z \neq 0$ in eq. (3.10). Instead, the contact terms in $C_{1}^{(S)}$ could not be skipped, because $C_{1}^{(S)}$ is integrated over the whole space-time.

We have demonstrated that the finite contact term to the order of $g^{0}$ in $C_{1}^{(S)}$ in the r.h.s. of eq. (3.10) is crucial to match the first term in the l.h.s. perturbatively (section 4). Thus, somehow surprisingly, the rationale for its occurrence is the low-energy theorem.

We have also observed that the $O\left(g^{4}\right)$ log-divergent contact term in $C_{1}^{(S)}$ [11-13] mixes in eq. (3.10) with the scheme-dependent divergences to the order of $g^{4}$ due to the second coefficient of the anomalous dimension of $F^{2}$ (section 4). Since the latter divergences are affected by the finite ambiguities to the order of $g^{2}$ (subsection 3.1), presently we cannot argue on the basis of the low-energy theorem about the renormalization of the $O\left(g^{4}\right) \log$ divergent contact term in $C_{1}^{(S)}$ perturbatively.

We have anyway computed the constant finite parts to the order of $g^{4}$ in both sides of eq. (3.10), with the IR subtraction point of the integral in the r.h.s. specified in section 4, for future employment as well.

Indeed, we have found in subsection 4.1 the prescription for the IR subtraction point of the integral in the r.h.s. of eq. (3.10) that also allows us to reproduce the finite small- $z$ dependence in the l.h.s. in perturbation theory to the order of $g^{2}$. 
Finally, in a massless QCD-like theory, by inverting the logic of the computation we have derived in section 6 from the low-energy theorem for the 2-point correlator, $\langle O(x) O(0)\rangle$, of a multiplicatively renormalizable gauge-invariant operator, $O$, the perturbative OPE coefficient, $C_{O}^{\left(F^{2}, O\right)}$, to the order of $g^{2} \cdot{ }^{6}$

\subsection{Low-energy theorem and nonperturbative UV asymptotics}

The aforementioned IR prescription, which also reproduces the $z$ dependence of the finite parts, has played a crucial role to verify the low-energy theorem nonperturbatively asymptotically in the UV.

Indeed, by skipping the divergent contact term in $C_{1}^{(S)}$, but not the finite one, we have verified the low-energy theorem (section 5) - eq. (1.2) for $n=2$ and $\mathcal{O}_{k}=F^{2}$ nonperturbatively in its canonical version - eq. (1.4) - by means of the renormalized RG-improved OPE asymptotically in the UV (appendices A and B). This is compatible with the previous result in [13], obtained independently of the low-energy theorem, that the divergent contact term in $C_{1}^{(S)}$ should be renormalized to zero.

Incidentally, for future applications, by working out the nonperturbative UV asymptotics of the OPE for $F^{2}$, we have computed the scale-dependent corrections to the universal asymptotics of the 2-point correlator of $\frac{g^{2}}{2 N} F^{2}$ (appendix B.3) - the YM Lagrangian density with the canonical normalization (subsection 2.3), whose correlators coincide with the correlators of the Wilson plaquette on the lattice in the continuum limit (subsection 2.3) - which is not RG invariant.

We have also computed the universal UV asymptotics of the 2-point correlator of its RG-invariant version, $\left(\frac{\beta(g)}{g}\right) F^{2}$ (section 5 and appendix B.3) that coincides (section 5), but for the overall scale-dependent normalization, with the UV asymptotics of the 2-point correlators of $\frac{g^{2}}{2} F^{2}$ and $F^{2}$ (appendix B.3).

Finally, by inverting the logic of the computation, we have derived from the low-energy theorem for any 2-point correlator, $\langle O(x) O(0)\rangle$, of a multiplicatively renormalizable gaugeinvariant operator, $O$, the nonperturbative UV asymptotics in section 6 by means of the lower-order perturbative OPE discussed above.

Since the r.h.s. of the low-energy theorem contains an operator insertion at zero momentum, it is somehow surprising that the low-energy theorem is asymptotically verified nonperturbatively in the UV.

\footnotetext{
${ }^{6}$ After the present paper has been posted in arXiv we have become aware of [27], where, interestingly, it has been shown how to obtain in principle the perturbative OPE coefficients directly in their renormalized form on the basis of an already renormalized analog — in the $\phi^{4}$ theory — of eq. (1.1) that has been derived independently of the present paper in the framework of a suitable BPHZ-like renormalization scheme, which does not employ explicitly the functional integral. Yet, the computations are very hard in the aforementioned scheme, and presently no explicit computation has been performed in YM theories [28], which a renormalized analog of eq. (1.1) applies to as well [28].This is to be contrasted with the approach in the present paper, where eq. (1.1) holds for the bare correlators and allows us an a priori evaluation of the log divergences from the anomalous dimensions, so that the OPE coefficients that produce the log divergences are easily evaluated. Moreover, the nonperturbative version of the low-energy theorem in terms of $\Lambda_{\mathrm{QCD}}$ in eq. (1.2) appears to be new. We would like to thank M.B. Frob for pointing out to us [27, 28] and for several discussions about his and our work.
} 
Yet, our RG-improved computations in sections 5 and 6 seem to show a posteriori that the integral on space-time in r.h.s. of the low-energy theorem, given the aforementioned specific choice of the IR subtraction point dictated by perturbation theory to the order of $g^{2}$, is in fact dominated by the UV asymptotics of the integrand. This is compatible with the universal belief that, nonperturbatively, the IR of the integrand in the r.h.s. of eqs. (3.10) and (6.6) is exponentially suppressed because of the glueball mass gap [3].

\section{A Nonperturbative UV asymptotics of the OPE}

According to the RG, the nonperturbative UV asymptotics of the renormalized OPE coefficients, $C_{0}, C_{1}$ and $C_{O}^{\left(O_{1}, O\right)}$, for multiplicatively renormalizable operators, $O$ and $O_{1}$ :

$$
\begin{aligned}
O(x) O(0) & \sim C_{0}^{(O)}(x) \mathbb{I}+C_{1}^{(O)}(x) O_{1}(0)+\cdots \\
O_{1}(x) O(0) & \sim C_{O}^{\left(O_{1}, O\right)}(x) O(0)+\cdots
\end{aligned}
$$

follows from the associated CS equations in the coordinate representation, which guarantees the absence of contact terms for $x \neq 0$ and, consequently, of additive renormalizations [15, $16,25]$ :

$$
\begin{aligned}
\left(x \cdot \frac{\partial}{\partial x}+\beta(g) \frac{\partial}{\partial g}+2 D+2 \gamma_{O}(g)\right) C_{0}^{(O)}(x) & =0 \\
\left(x \cdot \frac{\partial}{\partial x}+\beta(g) \frac{\partial}{\partial g}+2 D-D_{1}+2 \gamma_{O}(g)-\gamma_{O_{1}}(g)\right) C_{1}^{(O)}(x) & =0 \\
\left(x \cdot \frac{\partial}{\partial x}+\beta(g) \frac{\partial}{\partial g}+D_{1}+\gamma_{O_{1}}(g)\right) C_{O}^{\left(O_{1}, O\right)}(x) & =0
\end{aligned}
$$

with $D, \gamma_{O}(g)$ and $D_{1}, \gamma_{O_{1}}(g)$ the canonical and anomalous dimension of the operators $O$ and $O_{1}$ respectively.

The general solutions $[15,16,25]$ are:

$$
\begin{aligned}
C_{0}^{(O)}(x) & =\frac{1}{x^{2 D}} \mathcal{G}_{0}^{(O)}(g(x)) Z^{(O) 2}(x \mu, g(\mu)) \\
C_{1}^{(O)}(x) & =\frac{1}{x^{2 D-D_{1}}} \mathcal{G}_{1}^{(O)}(g(x)) Z^{(O) 2}(x \mu, g(\mu)) Z^{\left(O_{1}\right)-1}(x \mu, g(\mu)) \\
C_{O}^{\left(O_{1}, O\right)}(x) & =\frac{1}{x^{D_{1}}} \mathcal{G}_{O}^{\left(O_{1}, O\right)}(g(x)) Z^{\left(O_{1}\right)}(x \mu, g(\mu))
\end{aligned}
$$

which are expressed in terms of the RG-invariant functions, $\mathcal{G}_{0}^{(O)}, \mathcal{G}_{1}^{(O)}$ and $\mathcal{G}_{O}^{\left(O_{1}, O\right)}$, of $g(x)$ only, and of the renormalized multiplicative factors, $Z^{(O)}$ :

$$
Z^{(O)}(x \mu, g(\mu))=\exp \int_{g(\mu)}^{g(x)} \frac{\gamma_{O}(g)}{\beta(g)} d g
$$

determined by the anomalous dimension, $\gamma_{O}(g)$ :

$$
\gamma_{O}(g)=-\frac{\partial \log Z^{(O)}}{\partial \log \mu}=-\gamma_{0}^{(O)} g^{2}-\gamma_{1}^{(O)} g^{4}+\cdots
$$


and the beta function, $\beta(g)$ :

$$
\beta(g)=\frac{\partial g}{\partial \log \mu}=-\beta_{0} g^{3}-\beta_{1} g^{5}+\cdots
$$

that can be computed in perturbation theory.

The asymptotic expansion of $Z^{(O)}$ for $x \rightarrow 0$ follows from eq. (A.9):

$$
Z^{(O)}(x \mu, g(\mu)) \sim\left(\frac{g(x)}{g(\mu)}\right)^{\frac{\gamma_{0}^{(O)}}{\beta_{0}}} \exp \left(\frac{\gamma_{1}^{(O)} \beta_{0}-\gamma_{0}^{(O)} \beta_{1}}{2 \beta_{0}^{2}}\left(g^{2}(x)-g^{2}(\mu)\right)+\cdots\right)
$$

where the dots represent a series in integer powers of $g^{2}(x)$ and $g^{2}(\mu)$ higher than 1 . By an abuse of notation we have set $g(x) \equiv g\left(x \Lambda_{\mathrm{QCD}}\right)$ and $g(\mu) \equiv g\left(\mu^{-1} \Lambda_{\mathrm{QCD}}\right)$, where within the universal - i.e., renormalization-scheme independent - leading and next to leading asymptotic accuracy:

$$
g^{2}\left(x \Lambda_{\mathrm{QCD}}\right) \sim \frac{1}{\beta_{0} \log \left(\frac{1}{x^{2} \Lambda_{\mathrm{QCD}}^{2}}\right)}\left(1-\frac{\beta_{1}}{\beta_{0}^{2}} \frac{\log \log \left(\frac{1}{x^{2} \Lambda_{\mathrm{QCD}}^{2}}\right)}{\log \left(\frac{1}{x^{2} \Lambda_{\mathrm{QCD}}^{2}}\right)}\right)
$$

Indeed, in eq. (A.13) we may substitute to $\Lambda_{\mathrm{QCD}}$ any finite scale without changing the universal asymptotics. Thus:

$$
Z^{(O)}(x \mu, g(\mu)) \sim\left(\frac{g(x)}{g(\mu)}\right)^{\frac{\gamma_{0}^{(O)}}{\beta_{0}}} Z^{(O)^{\prime}}(g(\mu))
$$

where the constant factor, $Z^{(O)^{\prime}}(g(\mu))$, is the limit of the exponential in eq. (A.12) as $g(x) \rightarrow 0$. For brevity, in writing the universal UV asymptotics of $Z^{(O)}$, we skip systematically the factor of $Z^{(O)^{\prime}}(g(\mu))$, which is on the order of $1+O\left(g^{2}(\mu)\right)$.

Hence, the universal UV asymptotics of the OPE coefficients $[15,16,25]$ is:

$$
\begin{aligned}
C_{0}^{(O)}(x) & \sim \frac{1}{x^{2 D}}\left(\frac{g(x)}{g(\mu)}\right)^{\frac{2 \gamma_{0}^{(O)}}{\beta_{0}}} \\
C_{1}^{(O)}(x) & \sim \frac{1}{x^{2 D-D_{1}}} g^{2 l}(x)\left(\frac{g(x)}{g(\mu)}\right)^{\frac{2 \gamma_{0}^{(O)}-\gamma_{0}^{\left(O_{1}\right)}}{\beta_{0}}} \\
C_{O}^{\left(O_{1}, O\right)}(x) & \sim \frac{1}{x^{D_{1}}} g^{2 k}(x)\left(\frac{g(x)}{g(\mu)}\right)^{\frac{\left.\gamma_{0}^{(O}\right)}{\beta_{0}}}
\end{aligned}
$$

for some integer $l$ and $k$, up to constant normalization factors that can be fixed by perturbation theory.

The RG-invariant factors, $g^{2 l}(x)$ and $g^{2 k}(x)$, which arise from the asymptotics of $\mathcal{G}_{1}^{(O)}(g(x))$ and $\mathcal{G}_{O}^{\left(O_{1}, O\right)}(g(x))$ respectively, account for the possibility that the 3 -point correlator, $\left\langle O(z) O(0) O_{1}(x)\right\rangle$, vanishes to some perturbative order. 
Indeed, we have shown in section 6 that $\left\langle O(z) O(0) F^{2}(x)\right\rangle$ necessarily starts to the order of $g^{2}$ in perturbation theory up to contact terms as a consequence of the low-energy theorem, and thus actually vanishes to the lowest order up to contact terms.

Instead, the 2-point correlator, $C_{0}^{(O)}(x)$, of a nontrivial Hermitian operator, $O$, is necessarily nonvanishing $[15,16,25]$ in the conformal limit of a unitary massless AF QCDlike theory.

It follows from eqs. (4.9):

$$
C_{0}^{(S)}(x) \sim \frac{N^{2}-1}{\pi^{4} x^{8}}\left(\frac{g(x)}{g(\mu)}\right)^{4}
$$

from eq. (4.10):

$$
C_{1}^{(S)}(x) \sim \frac{4 \beta_{0}}{\pi^{2} x^{4}} g^{2}(x)\left(\frac{g(x)}{g(\mu)}\right)^{2}
$$

and from eq. (6.10):

$$
C_{O}^{\left(F^{2}, O\right)}(x) \sim \frac{2 \gamma_{0}^{(O)}}{\pi^{2} x^{4}} g^{2}(x)\left(\frac{g(x)}{g(\mu)}\right)^{2}
$$

\section{B Perturbative OPE}

\section{B.1 Perturbative $C_{0}^{(S)}$ in the momentum representation}

For the reader convenience, we report the relative normalization of the OPE coefficients computed in [11-13] for the multiplicatively renormalized operator, $F^{2}$, in the $\overline{M S}$ scheme:

$$
F^{2}(z) F^{2}(0) \sim 16 C_{0 C Z}^{(S)}(z) \mathbb{I}-4 C_{1 C Z}^{(S)}(z) F^{2}(0)
$$

with respect to our conventions:

$$
F^{2}(z) F^{2}(0) \sim C_{0}^{(S)}(z) \mathbb{I}+C_{1}^{(S)}(z) F^{2}(0)
$$

Thus:

$$
\begin{aligned}
& C_{0}^{(S)}=16 C_{0 C Z}^{(S)} \\
& C_{1}^{(S)}=-4 C_{1 C Z}^{(S)}
\end{aligned}
$$


$C_{0 C Z}^{(S)}$ has been computed in [11-13] to three loops in the momentum representation:

$$
\begin{aligned}
C_{0 C Z}^{(S)}(p)= & \frac{N^{2}-1}{16 \pi^{2}} p^{4}\left\{-\frac{\log \left(\frac{p^{2}}{\mu^{2}}\right)}{4}+\frac{1}{4}\right. \\
& +a_{s}\left(\frac{11}{48} C_{A} \log ^{2}\left(\frac{p^{2}}{\mu^{2}}\right)-\frac{73 C_{A} \log \left(\frac{p^{2}}{\mu^{2}}\right)}{48}-\frac{3 C_{A} \zeta_{3}}{4}+\frac{485 C_{A}}{192}\right. \\
& \left.-\frac{1}{12} \log ^{2}\left(\frac{p^{2}}{\mu^{2}}\right) N_{f} T_{F}+\frac{7}{12} \log \left(\frac{p^{2}}{\mu^{2}}\right) N_{f} T_{F}-\frac{17 N_{f} T_{F}}{16}\right) \\
& +a_{s}^{2}\left(-\frac{121}{576} C_{A}^{2} \log ^{3}\left(\frac{p^{2}}{\mu^{2}}\right)+\frac{313}{128} C_{A}^{2} \log ^{2}\left(\frac{p^{2}}{\mu^{2}}\right)+\frac{55}{32} C_{A}^{2} \log \left(\frac{p^{2}}{\mu^{2}}\right) \zeta_{3}\right. \\
& -\frac{37631 C_{A}^{2} \log \left(\frac{p^{2}}{\mu^{2}}\right)}{3456}-\frac{2059}{288} C_{A}^{2} \zeta_{3}+\frac{11}{64} C_{A}^{2} \zeta_{4}+\frac{25}{16} C_{A}^{2} \zeta_{5}+\frac{707201 C_{A}^{2}}{41472} \\
& +\frac{11}{72} C_{A} \log \left(\frac{p^{2}}{\mu^{2}}\right) N_{f} T_{F}-\frac{85}{48} C_{A} \log ^{2}\left(\frac{p^{2}}{\mu^{2}}\right) N_{f} T_{F}+\frac{1}{8} C_{A} \log \left(\frac{p^{2}}{\mu^{2}}\right) N_{f} T_{F} \zeta_{3} \\
& +\frac{6665}{864} C_{A} \log \left(\frac{p^{2}}{\mu^{2}}\right) N_{f} T_{F}+\frac{169}{144} C_{A} N_{f} T_{F} \zeta_{3}-\frac{7}{16} C_{A} N_{f} T_{F} \zeta_{4} \\
& -\frac{7847}{648} C_{A} N_{f} T_{F}-\frac{1}{8} C_{F} \log ^{2}\left(\frac{p^{2}}{\mu^{2}}\right) N_{f} T_{F}-\frac{3}{4} C_{F} \log \left(\frac{p^{2}}{\mu^{2}}\right) N_{f} T_{F} \zeta_{3} \\
& +\frac{131}{96} C_{F} \log \left(\frac{p^{2}}{\mu^{2}}\right) N_{f} T_{F}+\frac{41}{24} C_{F} N_{f} T_{F} \zeta_{3}+\frac{3}{8} C_{F} N_{f} T_{F} \zeta_{4} \\
& -\frac{5281 C_{F} N_{f} T_{F}}{1728}-\frac{1}{36} \log ^{3}\left(\frac{p^{2}}{\mu^{2}}\right) N_{f}^{2} T_{F}^{2}+\frac{7}{24} \log { }^{2}\left(\frac{p^{2}}{\mu^{2}}\right) N_{f}^{2} T_{F}^{2} \\
& \left.\left.-\frac{127}{108} \log \left(\frac{p^{2}}{\mu^{2}}\right) N_{f}^{2} T_{F}^{2}+\frac{4715 N_{f}^{2} T_{F}^{2}}{2592}\right)\right\}
\end{aligned}
$$

with $C_{A}=N, C_{F}=\frac{N^{2}-1}{2 N}, T_{F}=\frac{1}{2}$ and $a_{s}=\frac{g_{Y M}^{2}(\mu)}{4 \pi^{2}}$.

Hence, multiplying eq. (B.5) by 16, and expressing $a_{s}$ in terms of the 't Hooft coupling, $g$, we get $C_{0}^{(S)}$ in the momentum representation:

$$
\begin{aligned}
C_{0}^{(S)}(p)= & \frac{N^{2}-1}{4 \pi^{2}} p^{4}\left(1-\log \left(\frac{p^{2}}{\mu^{2}}\right)+g^{2}(\mu)\left(B_{0,1}^{(S)}-B_{0,2}^{(S)} \log \left(\frac{p^{2}}{\mu^{2}}\right)+\beta_{0} \log ^{2}\left(\frac{p^{2}}{\mu^{2}}\right)\right)\right. \\
& \left.+g^{4}(\mu)\left(B_{0,3}^{(S)}-B_{0,4}^{(S)} \log \left(\frac{p^{2}}{\mu^{2}}\right)+B_{0,5}^{(S)} \log ^{2}\left(\frac{p^{2}}{\mu^{2}}\right)+\beta_{0}^{2} \log ^{3}\left(\frac{p^{2}}{\mu^{2}}\right)\right)\right) \\
& +\frac{N^{2}-1}{4 \pi^{2}} p^{4}\left(\log \left(\frac{\Lambda^{2}}{\mu^{2}}\right)+g^{2}(\mu)\left(B_{0,6}^{(S)} \log \left(\frac{\Lambda^{2}}{\mu^{2}}\right)-\beta_{0} \log ^{2}\left(\frac{\Lambda^{2}}{\mu^{2}}\right)\right)\right. \\
& \left.+g^{4}(\mu)\left(B_{0,7}^{(S)} \log \left(\frac{\Lambda^{2}}{\mu^{2}}\right)+B_{0,8}^{(S)} \log ^{2}\left(\frac{\Lambda^{2}}{\mu^{2}}\right)+\beta_{0}^{2} \log ^{3}\left(\frac{\Lambda^{2}}{\mu^{2}}\right)\right)\right)
\end{aligned}
$$


with:

$$
\begin{aligned}
B_{0,1}^{(S)}= & \frac{1}{(4 \pi)^{2}}\left(\frac{485}{12}-12 \zeta_{3}-\frac{17}{2} \frac{N_{f}}{N}\right) \\
B_{0,2}^{(S)}= & \frac{1}{(4 \pi)^{2}}\left(\frac{73}{3}-\frac{14}{3} \frac{N_{f}}{N}\right) \\
B_{0,3}^{(S)}= & \frac{1}{(4 \pi)^{4}}\left(11 \zeta_{4}+100 \zeta_{5}-\frac{4118 \zeta_{3}}{9}+\frac{707201}{648}+\frac{584 N_{f} \zeta_{3}}{9 N}\right. \\
& \left.-\frac{141395 N_{f}}{324 N}+\frac{4715 N_{f}^{2}}{162 N^{2}}-\frac{6 N_{f} \zeta_{4}}{N^{3}}-\frac{82 N_{f} \zeta_{3}}{3 N^{3}}+\frac{5281 N_{f}}{108 N^{3}}\right) \\
B_{0,4}^{(S)}= & \frac{1}{(4 \pi)^{4}}\left(-110 \zeta_{3}+\frac{37631}{54}-4 \zeta_{3} \frac{N_{f}}{N}-\frac{6665}{27} \frac{N_{f}}{N}+24 \zeta_{3} \frac{N^{2}-1}{2 N^{2}} \frac{N_{f}}{N}\right. \\
& \left.-\frac{131}{3} \frac{N^{2}-1}{2 N^{2}} \frac{N_{f}}{N}+\frac{508}{27} \frac{N_{f}^{2}}{N^{2}}\right) \\
B_{0,5}^{(S)}= & \frac{1}{(4 \pi)^{4}}\left(\frac{313}{2}-\frac{170}{3} \frac{N_{f}}{N}-4 \frac{N^{2}-1}{2 N^{2}} \frac{N_{f}}{N}+\frac{14}{3} \frac{N_{f}^{2}}{N^{2}}\right) \\
B_{0,6}^{(S)}= & \frac{1}{(4 \pi)^{2}}\left(\frac{17}{2}-\frac{5}{3} \frac{N_{f}}{N}\right) \\
B_{0,7}^{(S)}= & \frac{1}{(4 \pi)^{4}}\left(\frac{22 \zeta_{3}}{3}+\frac{22351}{324}-\frac{28 \zeta_{3}}{3} \frac{N_{f}}{N}-\frac{1598}{81} \frac{N_{f}}{N}+8 \zeta_{3} \frac{N^{2}-1}{2 N^{2}}\right. \\
& \left.-\frac{107}{9} \frac{N^{2}-1}{2 N^{2}} \frac{N_{f}}{N}+\frac{49}{81} \frac{N_{f}^{2}}{N^{2}}\right) \\
B_{0,8}^{(S)}= & \frac{1}{(4 \pi)^{4}}\left(-\frac{833}{18}+\frac{146}{9} \frac{N_{f}}{N}+\frac{8}{3} \frac{N^{2}-1}{2 N^{2}} \frac{N_{f}}{N}-\frac{10}{9} \frac{N_{f}^{2}}{N^{2}}\right)
\end{aligned}
$$

In eq. (B.6) we have also included the contact terms computed in [13].

\section{B.2 Perturbative $C_{0}^{(S)}$ in the coordinate representation}

To get $C_{0}^{(S)}$ in the coordinate representation, we perform the Fourier transform employing the relations [15]:

$$
\begin{aligned}
\int p^{4} \log \frac{p^{2}}{\mu^{2}} e^{i p \cdot x} \frac{d^{4} p}{(2 \pi)^{4}}= & -\frac{2^{6} 3}{\pi^{2} x^{8}} \\
\int p^{4} \log ^{2} \frac{p^{2}}{\mu^{2}} e^{i \cdot x} \frac{d^{4} p}{(2 \pi)^{4}}= & \frac{2^{7} 3}{\pi^{2} x^{8}}\left(\log \left(\mu^{2} x^{2}\right)-\frac{10}{3}+2 \gamma_{E}-\log 4\right) \\
\int p^{4} \log ^{3} \frac{p^{2}}{\mu^{2}} e^{i p \cdot x} \frac{d^{4} p}{(2 \pi)^{4}}= & \frac{2^{6} 3}{\pi^{2} x^{8}}\left(-3\left(\log 4-\log \left(\mu^{2} x^{2}\right)\right)^{2}\right. \\
& +\left(20-12 \gamma_{E}\right) \log \left(\mu^{2} x^{2}\right)-12 \gamma_{E}^{2} \\
& \left.-\frac{51}{2}+40 \gamma_{E}-\left(20-12 \gamma_{E}\right) \log 4\right)
\end{aligned}
$$


It follows $C_{0}^{(S)}$ in the coordinate representation:

$$
\begin{aligned}
C_{0}^{(S)}(x)= & \frac{N^{2}-1}{x^{8}} \frac{48}{\pi^{4}}\left(1+g^{2}(\mu)\left(A_{0,1}^{(S)}+2 \beta_{0} \log \left(x^{2} \mu^{2}\right)\right)\right. \\
& \left.+g^{4}(\mu)\left(A_{0,2}^{(S)}+A_{0,3}^{(S)} \log \left(x^{2} \mu^{2}\right)+3 \beta_{0}^{2} \log ^{2}\left(x^{2} \mu^{2}\right)\right)\right) \\
+ & \Delta^{2} \delta^{(4)}(x) \frac{N^{2}-1}{4 \pi^{2}}\left(1+\log \left(\frac{\Lambda^{2}}{\mu^{2}}\right)+g^{2}(\mu)\left(A_{0,4}^{(S)}+A_{0,5}^{(S)} \log \left(\frac{\Lambda^{2}}{\mu^{2}}\right)\right.\right. \\
& \left.-\beta_{0} \log ^{2}\left(\frac{\Lambda^{2}}{\mu^{2}}\right)\right)+g^{4}(\mu)\left(A_{0,6}^{(S)}+A_{0,7}^{(S)} \log \left(\frac{\Lambda^{2}}{\mu^{2}}\right)+A_{0,8}^{(S)} \log ^{2}\left(\frac{\Lambda^{2}}{\mu^{2}}\right)\right. \\
& \left.\left.+\beta_{0}^{2} \log ^{3}\left(\frac{\Lambda^{2}}{\mu^{2}}\right)\right)\right)
\end{aligned}
$$

with $A_{0,1}^{(S)}, A_{0,2}^{(S)}, A_{0,3}^{(S)}$ scheme dependent:

$$
\begin{aligned}
A_{0,1}^{(S)}= & \frac{1}{(4 \pi)^{2}}\left(\frac{132 \gamma_{E}-1-132 \log 2}{9}-\frac{2 N_{f}\left(12 \gamma_{E}+1-12 \log 2\right)}{9 N}\right) \\
A_{0,2}^{(S)}= & \frac{1}{(4 \pi)^{4}}\left(\frac{-98+4356 \log ^{2} 2-8712 \gamma_{E} \log 2-2382 \log 2}{27}\right. \\
& +\frac{-2970 \zeta_{3}+4356 \gamma_{E}^{2}+2382 \gamma_{E}}{27} \\
& -\frac{N_{f}\left(-2112 \log 2-6336 \gamma_{E} \log 2\right)}{54 N} \\
& -\frac{N_{f}\left(-432 \zeta_{3}+3168 \gamma_{E}^{2}+2112 \gamma_{E}+121+3168 \log ^{2} 2\right)}{54 N} \\
& +\frac{2 N_{f}^{2}\left(72 \gamma_{E}^{2}+12 \gamma_{E}-13+72 \log 2-144 \gamma_{E} \log 2-12 \log 2\right)}{27 N^{2}} \\
& \left.+\frac{N_{f}\left(-24 \zeta_{3}+16 \gamma_{E}+17-16 \log 2\right)}{2 N^{3}}\right) \\
A_{0,3}^{(S)}= & \frac{1}{(4 \pi)^{4}}\left(\frac{1452 \gamma_{E}+397-1452 \log 2}{9}-\frac{88 N_{f}\left(6 \gamma_{E}+2-6 \log 2\right)}{9 N}\right. \\
& \left.+\frac{4 N_{f}^{2}\left(12 \gamma_{E}+1-12 \log 2\right)}{9 N^{2}}+\frac{4 N_{f}}{N^{3}}\right)
\end{aligned}
$$

and $A_{0,4}^{(S)}, A_{0,5}^{(S)}, A_{0,6}^{(S)}, A_{0,7}^{(S)}, A_{0,8}^{(S)}$ coefficients of the contact terms:

$$
\begin{aligned}
A_{0,4}^{(S)}= & \frac{1}{(4 \pi)^{2}}\left(\frac{485}{12}-12 \zeta_{3}-\frac{17 N_{f}}{2 N}\right) \\
A_{0,5}^{(S)}= & \frac{1}{(4 \pi)^{2}}\left(\frac{17}{2}-\frac{5}{3} \frac{N_{f}}{N}\right) \\
A_{0,6}^{(S)}= & \frac{1}{(4 \pi)^{4}}\left(11 \zeta_{4}+100 \zeta_{5}-\frac{4118 \zeta_{3}}{9}+\frac{707201}{648}+\frac{584 N_{f} \zeta_{3}}{9 N}\right. \\
& \left.-\frac{141395 N_{f}}{324 N}+\frac{4715 N_{f}^{2}}{162 N^{2}}-\frac{6 N_{f} \zeta_{4}}{N^{3}}-\frac{82 N_{f} \zeta_{3}}{3 N^{3}}+\frac{5281 N_{f}}{108 N^{3}}\right)
\end{aligned}
$$




$$
\begin{aligned}
A_{0,7}^{(S)}= & \frac{1}{(4 \pi)^{4}}\left(\frac{22 \zeta_{3}}{3}+\frac{22351}{324}-\frac{28 \zeta_{3}}{3} \frac{N_{f}}{N}-\frac{1598}{81} \frac{N_{f}}{N}+8 \zeta_{3} \frac{N^{2}-1}{2 N^{2}}\right. \\
& \left.-\frac{107}{9} \frac{N^{2}-1}{2 N^{2}} \frac{N_{f}}{N}+\frac{49}{81} \frac{N_{f}^{2}}{N^{2}}\right) \\
A_{0,8}^{(S)}= & \frac{1}{(4 \pi)^{4}}\left(-\frac{833}{18}+\frac{146}{9} \frac{N_{f}}{N}+\frac{8}{3} \frac{N^{2}-1}{2 N^{2}} \frac{N_{f}}{N}-\frac{10}{9} \frac{N_{f}^{2}}{N^{2}}\right)
\end{aligned}
$$

\section{B.3 Verifying the UV asymptotics of $C_{0}^{(S)}$ by a change of renormalization scheme in perturbation theory}

We skip the contact terms, and we multiply eq. (B.18) by $g^{4}$ that is equivalent to consider the OPE of $g^{2} F^{2}$. Then, following [15] we change the renormalization scheme redefining the coupling constant:

$$
g_{a b}^{2}(\mu)=g^{2}(\mu)\left(1+a g^{2}(\mu)+b g^{4}(\mu)\right)
$$

with $a$ and $b$ such that the constant finite $\operatorname{parts}^{7}$ of $g^{4} C_{0}^{(S)}$ vanish to the order of $g^{8}$. Eq. (B.18) becomes:

$$
\begin{aligned}
g_{a b}^{4}(\mu) C_{0}^{(S)}(x)= & \frac{N^{2}-1}{\pi^{4}} \frac{48 g_{a b}^{4}(\mu)}{x^{8}}\left(1+g_{a b}^{2}(\mu)\left(A_{0,1}^{(S)}-2 a+2 \beta_{0} \log \left(x^{2} \mu^{2}\right)\right)\right. \\
& +g_{a b}^{4}(\mu)\left(A_{0,2}^{(S)}+5 a^{2}-2 b-3 a A_{0,1}^{(S)}\right. \\
& \left.\left.+\left(A_{0,3}^{(S)}+\frac{a\left(2 N_{f}-11 N\right)}{8 \pi^{2} N}\right) \log \left(x^{2} \mu^{2}\right)+3 \beta_{0}^{2} \log ^{2}\left(x^{2} \mu^{2}\right)\right)\right)
\end{aligned}
$$

with:

$$
\begin{aligned}
& a=\frac{A_{0,1}^{(S)}}{2} \\
& b=\frac{4 A_{0,2}^{(S)}-A_{0,1}^{(S) 2}}{8}
\end{aligned}
$$

Remarkably, the coefficient of the term $g_{a b}^{4}(\mu) \log \left(x^{2} \mu^{2}\right)$ is [15] now:

$$
\begin{aligned}
& A_{0,3}^{(S)}+\frac{a\left(2 N_{f}-11 N\right)}{8 \pi^{2} N} \\
&=A_{0,3}^{(S)}+\frac{A_{0,1}^{(S)}\left(2 N_{f}-11 N\right)}{16 \pi^{2} N} \\
&=\frac{1}{(4 \pi)^{4}}\left(\frac{1452 \gamma_{E}+397-1452 \log 2}{9}-\frac{88 N_{f}\left(6 \gamma_{E}+2-6 \log 2\right)}{9 N}\right. \\
&\left.+\frac{4 N_{f}^{2}\left(12 \gamma_{E}+1-12 \log 2\right)}{9 N^{2}}+\frac{4 N_{f}}{N^{3}}\right) \\
&+\frac{\left(2 N_{f}-11 N\right)}{16 \pi^{2} N} \frac{1}{(4 \pi)^{2}}\left(\frac{132 \gamma_{E}-1-132 \log 2}{9}-\frac{2 N_{f}\left(12 \gamma_{E}+1-12 \log 2\right)}{9 N}\right) \\
&= \frac{34 N^{3}-13 N^{2} N_{f}+3 N_{f}}{192 \pi^{4} N^{3}}=4 \beta_{1}
\end{aligned}
$$

\footnotetext{
${ }^{7}$ We define the divergent parts as the terms that, after setting $\mu=\Lambda$, become divergent as $\Lambda \rightarrow \infty$. The constant finite parts are the remaining constant terms.
} 
It follows:

$$
\begin{aligned}
g_{a b}^{4}(\mu) C_{0}^{(S)}(x)= & \frac{N^{2}-1}{\pi^{4}} \frac{48 g_{a b}^{4}(\mu)}{x^{8}}\left(1+g_{a b}^{2}(\mu) 2 \beta_{0} \log \left(x^{2} \mu^{2}\right)\right. \\
& \left.+g_{a b}^{4}(\mu)\left(4 \beta_{1} \log \left(x^{2} \mu^{2}\right)+3 \beta_{0}^{2} \log ^{2}\left(x^{2} \mu^{2}\right)\right)\right)
\end{aligned}
$$

Thus, manifestly $\gamma_{1}^{\left(F^{2}\right)}=4 \beta_{1}$ in this scheme, according to eq. (4.4).

In order to verify eq. (A.18) we should express eq. (B.32) in terms of $g(x)$, which reads to two loops in $\overline{M S}$-like schemes:

$$
\begin{aligned}
g^{2}(x)= & g^{2}(\mu)\left(1+g^{2}(\mu) \beta_{0} \log \left(x^{2} \mu^{2}\right)+g^{4}(\mu)\left(\beta_{1} \log \left(x^{2} \mu^{2}\right)\right.\right. \\
& \left.\left.+\beta_{0}^{2} \log ^{2}\left(x^{2} \mu^{2}\right)\right)\right)
\end{aligned}
$$

Hence, to two loops:

$$
\begin{aligned}
g^{4}(x)= & g^{4}(\mu)\left(1+g^{2}(\mu) 2 \beta_{0} \log \left(x^{2} \mu^{2}\right)+g^{4}(\mu)\left(2 \beta_{1} \log \left(x^{2} \mu^{2}\right)\right.\right. \\
& \left.\left.+3 \beta_{0}^{2} \log ^{2}\left(x^{2} \mu^{2}\right)\right)\right)
\end{aligned}
$$

This cannot be done immediately, because the coefficient of the term $g_{a b}^{4}(\mu) \log \left(x^{2} \mu^{2}\right)$ in eq. (B.32) is $4 \beta_{1}$ instead of $2 \beta_{1}$ in eq. (B.34). Following [15] we insert the identity:

$$
1=\frac{1+2 \frac{\beta_{1}}{\beta_{0}} g_{a b}^{2}(\mu)}{1+2 \frac{\beta_{1}}{\beta_{0}} g_{a b}^{2}(x)} \frac{1+2 \frac{\beta_{1}}{\beta_{0}} g_{a b}^{2}(x)}{1+2 \frac{\beta_{1}}{\beta_{0}} g_{a b}^{2}(\mu)}
$$

in eq. (B.32) to the relevant order:

$$
\begin{aligned}
g_{a b}^{4}(\mu) C_{0}^{(S)}(x)= & \frac{1+2 \frac{\beta_{1}}{\beta_{0}} g_{a b}^{2}(\mu)}{1+2 \frac{\beta_{1}}{\beta_{0}} g_{a b}^{2}(x)} \frac{1+2 \frac{\beta_{1}}{\beta_{0}} g_{a b}^{2}(x)}{1+2 \frac{\beta_{1}}{\beta_{0}} g_{a b}^{2}(\mu)} \frac{N^{2}-1}{\pi^{4}} \frac{48 g_{a b}^{4}(\mu)}{x^{8}} \\
& \left(1+g_{a b}^{2}(\mu) 2 \beta_{0} \log \left(x^{2} \mu^{2}\right)+g_{a b}^{4}(\mu)\left(4 \beta_{1} \log \left(x^{2} \mu^{2}\right)+3 \beta_{0}^{2} \log ^{2}\left(x^{2} \mu^{2}\right)\right)\right) \\
= & \frac{1+2 \frac{\beta_{1}}{\beta_{0}} g_{a b}^{2}(x)}{1+2 \frac{\beta_{1}}{\beta_{0}} g_{a b}^{2}(\mu)} \frac{N^{2}-1}{\pi^{4}} \frac{48 g_{a b}^{4}(\mu)}{x^{8}}\left(1+g_{a b}^{2}(\mu) 2 \beta_{0} \log \left(x^{2} \mu^{2}\right)\right. \\
& \left.+g_{a b}^{4}(\mu)\left(2 \beta_{1} \log \left(x^{2} \mu^{2}\right)+3 \beta_{0}^{2} \log ^{2}\left(x^{2} \mu^{2}\right)\right)\right) \\
= & \frac{N^{2}-1}{\pi^{4}} \frac{48}{x^{8}} g_{a b}^{4}(x)\left(1+2 \frac{\beta_{1}}{\beta_{0}} g_{a b}^{2}(x)-2 \frac{\beta_{1}}{\beta_{0}} g_{a b}^{2}(\mu)\right)
\end{aligned}
$$

where we have expanded to the order of $g^{4}$ :

$$
\frac{1+2 \frac{\beta_{1}}{\beta_{0}} g_{a b}^{2}(\mu)}{1+2 \frac{\beta_{1}}{\beta_{0}} g_{a b}^{2}(x)}=1-2 \beta_{1} g_{a b}^{4}(\mu) \log \left(x^{2} \mu^{2}\right)
$$

and to the order of $g^{2}$ :

$$
\frac{1+2 \frac{\beta_{1}}{\beta_{0}} g_{a b}^{2}(x)}{1+2 \frac{\beta_{1}}{\beta_{0}} g_{a b}^{2}(\mu)}=1+2 \frac{\beta_{1}}{\beta_{0}} g_{a b}^{2}(x)-2 \frac{\beta_{1}}{\beta_{0}} g_{a b}^{2}(\mu)
$$


The factor of $\left(1+2 \frac{\beta_{1}}{\beta_{0}} g_{a b}^{2}(x)-2 \frac{\beta_{1}}{\beta_{0}} g_{a b}^{2}(\mu)\right)$ in the last line of eq. (B.36) arises according to eq. (A.12), as it is verified by setting $\gamma_{0}^{\left(F^{2}\right)}=2 \beta_{0}$ and $\gamma_{1}^{\left(F^{2}\right)}=4 \beta_{1}$ in eq. (A.12):

$$
\begin{aligned}
& \left\langle\frac{g_{a b}^{2}(\mu)}{2} F^{2}(x) \frac{g_{a b}^{2}(\mu)}{2} F^{2}(0)\right\rangle \sim \frac{g_{a b}^{4}(\mu)}{4} C_{0}^{(S)}(x) \\
& \sim \frac{N^{2}-1}{\pi^{4}} \frac{12}{x^{8}} g_{a b}^{4}(\mu) Z^{\left(F^{2}\right) 2}\left(x \mu, g_{a b}(\mu)\right) \\
& \sim \frac{N^{2}-1}{\pi^{4}} \frac{12}{x^{8}} g_{a b}^{4}(\mu)\left(\frac{g_{a b}(x)}{g_{a b}(\mu)}\right)^{\frac{2 \gamma_{0}^{\left(F^{2}\right)}}{\beta_{0}}} \exp \left(\frac{\gamma_{1}^{\left(F^{2}\right)} \beta_{0}-\gamma_{0}^{\left(F^{2}\right)} \beta_{1}}{\beta_{0}^{2}}\left(g_{a b}^{2}(x)-g_{a b}^{2}(\mu)\right)+\cdots\right) \\
& \sim \frac{N^{2}-1}{\pi^{4}} \frac{12}{x^{8}} g_{a b}^{4}(x)\left(1+2 \frac{\beta_{1}}{\beta_{0}} g_{a b}^{2}(x)-2 \frac{\beta_{1}}{\beta_{0}} g_{a b}^{2}(\mu)+\cdots\right)
\end{aligned}
$$

Therefore, eq. (B.36) agrees with eq. (A.18).

The computation in eq. (B.39) exhibits the scale dependence in massless QCD of the 2-point correlator of $\frac{g^{2}}{2} F^{2}$ in the scheme where manifesly $\gamma_{1}^{\left(F^{2}\right)}=4 \beta_{1}$. Hence, the aforementioned operator is not RG invariant.

As an aside, $\frac{g^{2}}{2 N} F^{2}$ is the YM Lagrangian density with the canonical normalization (subsection 2.3).

We may remove the scale-dependent term, $-2 \frac{\beta_{1}}{\beta_{0}} g_{a b}^{2}(\mu)$, in the last line of eqs. (B.36) and (B.39) multiplying them by $\beta_{0}^{2}\left(1+\frac{\beta_{1}}{\beta_{0}} g_{a b}^{2}(\mu)\right)^{2}$ that makes $g_{a b}^{4}(\mu) C_{0}^{(S)}$ RG invariant to the relevant perturbative order [15]. Hence, we get:

$$
\begin{aligned}
\left(\frac{\beta(g)}{g}\right)^{2} C_{0}^{(S)}(x) & \sim \frac{N^{2}-1}{\pi^{4}} \frac{48 \beta_{0}^{2}}{x^{8}} g_{a b}^{4}(x)\left(1+2 \frac{\beta_{1}}{\beta_{0}} g_{a b}^{2}(x)\right) \\
& \sim \frac{N^{2}-1}{\pi^{4}} \frac{48 \beta_{0}^{2}}{x^{8}} g_{a b}^{4}(x)
\end{aligned}
$$

according to the nonperturbative asymptotics in eq. (5.1).

\section{B.4 Perturbative $C_{1}^{(S)}$ in the momentum representation}

$C_{1 C Z}^{(S)}$ has been computed in [11-13] to three loops in the momentum representation in the $\overline{M S}$ scheme:

$$
\begin{aligned}
C_{1 C Z}^{(S)}(p)=- & +a_{s}\left\{-\frac{49 C_{A}}{36}+\frac{5 N_{f} T_{F}}{9}-\log \left(\frac{p^{2}}{\mu^{2}}\right)\left(\frac{N_{f} T_{F}}{3}-\frac{11 C_{A}}{12}\right)\right\} \\
+ & a_{s}^{2}\left\{\frac{33 C_{A}^{2} \zeta_{3}}{8}-\frac{11509 C_{A}^{2}}{1296}+\frac{3}{2} C_{A} N_{f} T_{F} \zeta_{3}+\frac{3095 C_{A} N_{f} T_{F}}{648}-3 C_{F} N_{f} T_{F} \zeta_{3}\right. \\
& +\frac{13 C_{F} N_{f} T_{F}}{4}-\frac{25 N_{f}^{2} T_{F}^{2}}{81}-\log \left(\frac{p^{2}}{\mu^{2}}\right)\left(-\frac{1151 C_{A}^{2}}{216}+\frac{97 C_{A} N_{f} T_{F}}{27}+C_{F} N_{f} T_{F}\right. \\
& \left.-\frac{10 N_{f}^{2} T_{F}^{2}}{27}\right)+\log ^{2}\left(\frac{p^{2}}{\mu^{2}}\right)\left(-\frac{121 C_{A}^{2}}{144}+\frac{11 C_{A} N_{f} T_{F}}{18}-\frac{N_{f}^{2} T_{F}^{2}}{9}\right) \\
& \left.+\log \left(\frac{\Lambda^{2}}{\mu^{2}}\right)\left[-\frac{17 C_{A}^{2}}{24}+\frac{5 C_{A} N_{f} T_{F}}{12}+\frac{C_{F} N_{f} T_{F}}{4}\right]\right\}
\end{aligned}
$$




$$
\begin{aligned}
+ & a_{s}^{3}\left\{\frac{5315 C_{A}^{3} \zeta_{3}}{144}-\frac{55 C_{A}^{3} \zeta_{5}}{8}-\frac{9775633 C_{A}^{3}}{186624}-\frac{263}{144} C_{A}^{2} N_{f} T_{F} \zeta_{3}\right. \\
& -5 C_{A}^{2} N_{f} T_{F} \zeta_{5}+\frac{1299295 C_{A}^{2} N_{f} T_{F}}{31104}-\frac{331}{16} C_{A} C_{F} N_{f} T_{F} \zeta_{3}-\frac{15}{2} C_{A} C_{F} N_{f} T_{F} \zeta_{5} \\
& +\frac{35707 C_{A} C_{F} N_{f} T_{F}}{1152}-\frac{121}{36} C_{A} N_{f}^{2} T_{F}^{2} \zeta_{3}-\frac{116773 C_{A} N_{f}^{2} T_{F}^{2}}{15552}-9 C_{F}^{2} N_{f} T_{F} \zeta_{3} \\
& +15 C_{F}^{2} N_{f} T_{F} \zeta_{5}-\frac{45}{16} C_{F}^{2} N_{f} T_{F}+\frac{13}{2} C_{F} N_{f}^{2} T_{F}^{2} \zeta_{3}-\frac{2399}{288} C_{F} N_{f}^{2} T_{F}^{2}+\frac{125 N_{f}^{3} T_{F}^{3}}{729} \\
& -\log \left(\frac{p^{2}}{\mu^{2}}\right)\left(\frac{363 C_{A}^{3} \zeta_{3}}{32}-\frac{360325 C_{A}^{3}}{10368}+\frac{55757 C_{A}^{2} N_{f} T_{F}}{1728}-\frac{33}{4} C_{A} C_{F} N_{f} T_{F} \zeta_{3}\right. \\
& +\frac{2527}{192} C_{A} C_{F} N_{f} T_{F}-\frac{3}{2} C_{A} N_{f}^{2} T_{F}^{2} \zeta_{3}-\frac{2057}{288} C_{A} N_{f}^{2} T_{F}^{2}-\frac{9}{32} C_{F}^{2} N_{f} T_{F} \\
& \left.+3 C_{F} N_{f}^{2} T_{F}^{2} \zeta_{3}-\frac{209}{48} C_{F} N_{f}^{2} T_{F}^{2}+\frac{25 N_{f}^{3} T_{F}^{3}}{81}\right)+\log \left(\frac{p^{2}}{\mu^{2}}\right)\left(-\frac{1793 C_{A}^{3}}{216}\right. \\
& \left.+\frac{273}{32} C_{A}^{2} N_{f} T_{F}+\frac{55}{32} C_{A} C_{F} N_{f} T_{F}-\frac{181}{72} C_{A} N_{f}^{2} T_{F}^{2}-\frac{5}{8} C_{F} N_{f}^{2} T_{F}^{2}+\frac{5 N_{f}^{3} T_{F}^{3}}{27}\right) \\
& -\log 3\left(\frac{p^{2}}{\mu^{2}}\right)\left(-\frac{1331 C_{A}^{3}}{1728}+\frac{121}{144} C_{A}^{2} N_{f} T_{F}-\frac{11}{36} C_{A} N_{f}^{2} T_{F}^{2}+\frac{N_{f}^{3} T_{F}^{3}}{27}\right) \\
& +\log \left(\frac{\Lambda^{2}}{\mu^{2}}\right)\left[\frac{1415 C_{A}^{2} N_{f} T_{F}}{864}-\frac{2857 C_{A}^{3}}{1728}+\frac{205 C_{A} C_{F} N_{f} T_{F}}{288}-\frac{79 C_{A} N_{f}^{2} T_{F}^{2}}{432}\right. \\
& \left.-\frac{C_{F}^{2} N_{f} T_{F}}{16}-\frac{11 C_{F} N_{f}^{2} T_{F}^{2}}{72}\right]+\log ^{2}\left(\frac{\Lambda^{2}}{\mu^{2}}\right)\left[-\frac{89 C_{A}^{2} N_{f} T_{F}}{144}+\frac{187 C_{A}^{3}}{288}\right. \\
& \left.\left.-\frac{11 C_{A} C_{F} N_{f} T_{F}}{48}+\frac{5 C_{A} N_{f}^{2} T_{F}^{2}}{36}+\frac{C_{F} N_{f}^{2} T_{F}^{2}}{12}\right]\right\}
\end{aligned}
$$

It follows $C_{1}^{(S)}$ in the momentum representation:

$$
\begin{aligned}
C_{1}^{(S)}(p)= & 4+g^{2}(\mu)\left(B_{1,1}^{(S)}+\beta_{0} \log \left(\frac{p^{2}}{\mu^{2}}\right)\right)+g^{4}(\mu)\left(B_{1,2}^{(S)}+B_{1,3}^{(S)} \log \left(\frac{p^{2}}{\mu^{2}}\right)\right. \\
& \left.-\beta_{0}^{2} \log ^{2}\left(\frac{p^{2}}{\mu^{2}}\right)+4 \beta_{1} \log \left(\frac{\Lambda^{2}}{\mu^{2}}\right)\right)+g^{6}(\mu)\left(B_{1,4}^{(S)}+B_{1,5}^{(S)} \log \left(\frac{p^{2}}{\mu^{2}}\right)\right. \\
& \left.+B_{1,6}^{(S)} \log ^{2}\left(\frac{p^{2}}{\mu^{2}}\right)-\beta_{0}^{3} \log ^{3}\left(\frac{p^{2}}{\mu^{2}}\right)+8 \beta_{2} \log \left(\frac{\Lambda^{2}}{\mu^{2}}\right)-4 \beta_{0} \beta_{1} \log ^{2}\left(\frac{\Lambda^{2}}{\mu^{2}}\right)\right)
\end{aligned}
$$

with:

$$
\begin{aligned}
B_{1,1}^{(S)}= & \frac{1}{(4 \pi)^{2}}\left(\frac{196}{9}-\frac{40}{9} \frac{N_{f}}{N}\right) \\
B_{1,2}^{(S)}= & \frac{1}{(4 \pi)^{4}}\left(-\frac{264 \zeta_{3}}{2}+\frac{46036}{81}-\frac{12380}{81} \frac{N_{f}}{N}-48 \zeta_{3} \frac{N_{f}}{N}+\frac{8}{3} \zeta_{3} \frac{N^{2}-1}{2 N^{2}} \frac{N_{f}}{N}\right. \\
& \left.-104 \frac{N^{2}-1}{2 N^{2}} \frac{N_{f}}{N}+\frac{400}{81} \frac{N_{f}^{2}}{N^{2}}\right) \\
B_{1,3}^{(S)}= & \frac{1}{(4 \pi)^{4}}\left(-\frac{9208}{27}+\frac{3104}{27} \frac{N_{f}}{N}+16 \frac{N^{2}-1}{N^{2}} \frac{N_{f}}{N}-\frac{160}{27} \frac{N_{f}^{2}}{N^{2}}\right)
\end{aligned}
$$




$$
\begin{aligned}
B_{1,4}^{(S)}= & \frac{1}{(4 \pi)^{6}}\left(\frac{85040 \zeta_{3}}{9}-1760 \zeta_{5}-\frac{9775633}{729}-\frac{16612 N_{f} \zeta_{3}}{9 N}-\frac{640 N_{f} \zeta_{5}}{N}\right. \\
& +\frac{3518939 N_{f}}{486 N}-\frac{64 N_{f}^{2} \zeta_{3}}{9 N^{2}}-\frac{181546 N_{f}^{2}}{243 N^{2}}+\frac{1900 N_{f} \zeta_{3}}{N^{3}}-\frac{480 N_{f} \zeta_{5}}{N^{3}}-\frac{32467 N_{f}}{18 N^{3}} \\
& \left.+\frac{4000 N_{f}^{3}}{729 N^{3}}-\frac{52 N_{f}^{2} \zeta_{3}}{N^{4}}+\frac{2399 N_{f}^{2}}{9 N^{4}}-\frac{288 N_{f} \zeta_{3}}{N^{5}}+\frac{480 N_{f} \zeta_{5}}{N^{5}}-\frac{90 N_{f}}{N^{5}}\right) \\
B_{1,5}^{(S)}= & \frac{1}{(4 \pi)^{6}}\left(-2904 \zeta_{3}+\frac{720650}{81}+\frac{528 N_{f} \zeta_{3}}{N}-\frac{134014 N_{f}}{27 N}+\frac{5368 N_{f}^{2}}{9 N^{2}}\right. \\
& \left.-\frac{800 N_{f}^{3}}{81 N^{3}}-\frac{528 N_{f} \zeta_{3}}{N^{3}}+\frac{2473 N_{f}}{3 N^{3}}+\frac{96 N_{f}^{2} \zeta_{3}}{N^{4}}-\frac{418 N_{f}^{2}}{3 N^{4}}+\frac{9 N_{f}}{N^{5}}\right) \\
B_{1,6}^{(S)}= & \frac{1}{(4 \pi)^{6}}\left(\frac{57376}{27}-\frac{1202 N_{f}}{N}+\frac{1628 N_{f}^{2}}{9 N^{2}}-\frac{160 N_{f}^{3}}{27 N^{3}}+\frac{110 N_{f}}{N^{3}}-\frac{20 N_{f}^{2}}{N^{4}}\right)
\end{aligned}
$$

As demonstrated in [13], the divergent contact terms in eq. (B.42) are expressed in terms of the coefficients of the QCD beta function:

$$
\begin{aligned}
& \beta_{0}=\frac{1}{(4 \pi)^{2}}\left(\frac{11}{3}-\frac{2}{3} \frac{N_{f}}{N}\right) \\
& \beta_{1}=\frac{1}{(4 \pi)^{4}}\left(\frac{34}{3}-\frac{13}{3} \frac{N_{f}}{N}+\frac{N_{f}}{N^{3}}\right) \\
& \beta_{2}=\frac{1}{(4 \pi)^{6}}\left(\frac{2857}{54}-\frac{1709}{54} \frac{N_{f}}{N}+\frac{56}{27} \frac{N_{f}^{2}}{N^{2}}+\frac{187}{36} \frac{N_{f}}{N^{3}}-\frac{11}{18} \frac{N_{f}^{2}}{N^{4}}+\frac{N_{f}}{4 N^{5}}\right)
\end{aligned}
$$

\section{B.5 Perturbative $C_{1}^{(S)}$ in the coordinate representation}

The Fourier transform of eq. (B.41) is:

$$
\begin{aligned}
C_{1}^{(S)}(x)= & \frac{4 \beta_{0}}{\pi^{2} x^{4}} g^{2}(\mu)\left(1+g^{2}(\mu)\left(A_{1,1}^{(S)}+2 \beta_{0} \log \left(x^{2} \mu^{2}\right)\right)+g^{4}(\mu)\left(A_{1,2}^{(S)}\right.\right. \\
& \left.\left.+A_{1,3}^{(S)} \log \left(x^{2} \mu^{2}\right)+3 \beta_{0}^{2} \log ^{2}\left(x^{2} \mu^{2}\right)\right)\right)+\delta^{(4)}(x)\left(4+g^{2}(\mu) A_{1,4}^{(S)}\right. \\
& +g^{4}(\mu)\left(A_{1,5}^{(S)}+4 \beta_{1} \log \left(\frac{\Lambda^{2}}{\mu^{2}}\right)\right)+g^{6}(\mu)\left(A_{1,6}^{(S)}+8 \beta_{2} \log \left(\frac{\Lambda^{2}}{\mu^{2}}\right)\right. \\
& \left.\left.-4 \beta_{0} \beta_{1} \log ^{2}\left(\frac{\Lambda^{2}}{\mu^{2}}\right)\right)\right)
\end{aligned}
$$

with $A_{1,1}^{(S)}, A_{1,2}^{(S)}, A_{1,3}^{(S)}$ scheme dependent:

$$
\begin{aligned}
A_{1,1}^{(S)}= & \frac{4}{\beta_{0}} \frac{1}{(4 \pi)^{4}}\left(\frac{363 \gamma_{E}+394-363 \log 2}{27}-\frac{N_{f}\left(132 \gamma_{E}+155-132 \log 2\right)}{27 N}\right. \\
& \left.+\frac{4 N_{f}^{2}\left(3 \gamma_{E}+1-3 \log 2\right)}{27 N^{2}}+\frac{N_{f}}{N^{3}}\right)
\end{aligned}
$$




$$
\begin{aligned}
A_{1,2}^{(S)}= & \frac{16}{\beta_{0}} \frac{1}{(4 \pi)^{6}}\left(\frac{-117612 \zeta_{3}+95832 \gamma_{E}^{2}+248424 \gamma_{E}+188197+95832 \log ^{2} 2}{2592}\right. \\
& -\frac{191664 \gamma_{E} \log 2+248424 \log 2}{2592}-\frac{N_{f}\left(-7128 \zeta_{3}+17424 \gamma_{E}^{2}+47484 \gamma_{E}\right)}{864 N} \\
& +\frac{N_{f}\left(34553+17424 \log ^{2} 2-34848 \gamma_{E} \log 2-47484 \log 2\right)}{864 N} \\
& +\frac{11 N_{f}^{2}\left(4 \gamma_{E}+3-4 \log 2\right)\left(3 \gamma_{E}+4-3 \log 2\right)}{36 N^{2}}-\frac{1152 \gamma_{E}^{2} N_{f}^{3}}{5184 N^{3}} \\
& +\frac{N_{f}\left(+768 \gamma_{E} N_{f}^{2}-160 N_{f}^{2}-768 N_{f}^{2} \log 2+1152 N_{f}^{2} \log ^{2} 2\right)}{5184 N^{3}} \\
& +\frac{N_{f}\left(-2304 \gamma_{E} N_{f}^{2} \log 2+42768 \zeta_{3}-35640 \gamma_{E}-48951+35640 \log 2\right)}{5184 N^{3}} \\
& \left.-\frac{N_{f}^{2}\left(-144 \zeta_{3}+120 \gamma_{E}+149-120 \log 2\right)}{96 N^{4}}+\frac{9 N_{f}}{64 N^{5}}\right) \\
A_{1,3}^{(S)}= & \frac{16}{\beta_{0}} \frac{1}{(4 \pi)^{6}}\left(\frac{11\left(726 \gamma_{E}+941-726 \log 2\right)}{216}-\frac{N_{f}\left(968 \gamma_{E}+1319-968 \log 2\right)}{48 N}\right. \\
& +\frac{11 N_{f}^{2}\left(72 \gamma_{E}+75-48 \log (2)-24 \log 2\right)}{216 N^{2}} \\
& \left.+\frac{-96 \gamma_{E} N_{f}^{3}-32 N_{f}^{3}+96 N_{f}^{3} \log 2+1485 N_{f}}{432 N^{3}}-\frac{5 N_{f}^{2}}{8 N^{4}}\right)
\end{aligned}
$$

and $A_{1,4}^{(S)}, A_{1,5}^{(S)}, A_{1,6}^{(S)}$ coefficients of the contact terms:

$$
\begin{aligned}
A_{1,4}^{(S)}= & \frac{1}{(4 \pi)^{2}}\left(\frac{196}{9}-\frac{40}{9} \frac{N_{f}}{N}\right) \\
A_{1,5}^{(S)}= & \frac{1}{(4 \pi)^{4}}\left(-\frac{264 \zeta_{3}}{2}+\frac{46036}{81}-\frac{12380}{81} \frac{N_{f}}{N}-48 \zeta_{3} \frac{N_{f}}{N}+\frac{8}{3} \zeta_{3} \frac{N^{2}-1}{2 N^{2}} \frac{N_{f}}{N}\right. \\
& \left.-104 \frac{N^{2}-1}{2 N^{2}} \frac{N_{f}}{N}+\frac{400}{81} \frac{N_{f}^{2}}{N^{2}}\right) \\
A_{1,6}^{(S)}= & \frac{1}{(4 \pi)^{6}}\left(\frac{85040 \zeta_{3}}{9}-1760 \zeta_{5}-\frac{9775633}{729}-\frac{16612 N_{f} \zeta_{3}}{9 N}-\frac{640 N_{f} \zeta_{5}}{N}\right. \\
& +\frac{3518939 N_{f}}{486 N}-\frac{64 N_{f}^{2} \zeta_{3}}{9 N^{2}}-\frac{181546 N_{f}^{2}}{243 N^{2}}+\frac{1900 N_{f} \zeta_{3}}{N^{3}}-\frac{480 N_{f} \zeta_{5}}{N^{3}}-\frac{32467 N_{f}}{18 N^{3}} \\
& \left.+\frac{4000 N_{f}^{3}}{729 N^{3}}-\frac{52 N_{f}^{2} \zeta_{3}}{N^{4}}+\frac{2399 N_{f}^{2}}{9 N^{4}}-\frac{288 N_{f} \zeta_{3}}{N^{5}}+\frac{480 N_{f} \zeta_{5}}{N^{5}}-\frac{90 N_{f}}{N^{5}}\right)
\end{aligned}
$$

\section{B.6 Verifying the UV asymptotics of $C_{1}^{(S)}$ by a change of renormalization scheme in perturbation theory}

As for $C_{0}^{(S)}$, we skip the contact terms, and we multiply eq. (B.52) by $g^{2}$ that is equivalent to consider the OPE of $g^{2} F^{2}$. Following [15] we change the renormalization scheme redefining the coupling constant:

$$
g_{c d}^{2}(\mu)=g^{2}(\mu)\left(1+c g^{2}(\mu)+d g^{4}(\mu)\right)
$$


Then, eq. (B.52) becomes:

$$
\begin{aligned}
g_{c d}^{2}(\mu) C_{1}^{(S)}(x)= & \frac{4 \beta_{0}}{\pi^{2} x^{4}} g_{c d}^{4}(\mu)\left(1+g_{c d}^{2}(\mu)\left(A_{1,1}^{(S)}-2 c+2 \beta_{0} \log \left(x^{2} \mu^{2}\right)\right)\right. \\
& +g_{c d}^{4}(\mu)\left(5 c^{2}-2 d-3 c A_{1,1}^{(S)}+A_{1,2}^{(S)}\right. \\
& \left.+\left(A_{1,3}^{(S)}-6 c \beta_{0}\right) \log \left(x^{2} \mu^{2}\right)+3 \beta_{0} \log ^{2}\left(x^{2} \mu^{2}\right)\right)
\end{aligned}
$$

We fix $c$ and $d$ requiring that the finite parts of $g^{2} C_{1}^{(S)}$ vanish to the order of $g^{8}$ in the new renormalization scheme:

$$
\begin{aligned}
c & =\frac{A_{1,1}^{(S)}}{2} \\
d & =\frac{4 A_{1,2}^{(S)}-A_{1,1}^{(S) 2}}{8}
\end{aligned}
$$

Remarkably, as for $g_{a b}^{4} C_{0}^{(S)}$, in this renormalization scheme the coefficient of the term $g_{c d}^{4}(\mu) \log \left(x^{2} \mu^{2}\right)$ in eq. (B.60) is proportional to the second coefficient of the QCD beta function:

$$
\begin{aligned}
& A_{1,3}^{(S)}-6 c \beta_{0} \\
&=A_{1,3}^{(S)}-3 A_{1,1}^{(S)} \beta_{0} \\
&=\frac{16}{\beta_{0}} \frac{1}{(4 \pi)^{6}}\left(\frac{11\left(726 \gamma_{E}+941-726 \log 2\right)}{216}-\frac{N_{f}\left(968 \gamma_{E}+1319-968 \log 2\right)}{48 N}\right. \\
& \quad+\frac{11 N_{f}^{2}\left(72 \gamma_{E}+75-48 \log 2-24 \log 2\right)}{216 N^{2}} \\
&\left.+\frac{-96 \gamma_{E} N_{f}^{3}-32 N_{f}^{3}+96 N_{f}^{3} \log 2+1485 N_{f}}{432 N^{3}}-\frac{5 N_{f}^{2}}{8 N^{4}}\right) \\
& \\
&-\frac{12}{(4 \pi)^{4}}\left(\frac{363 \gamma_{E}+394-363 \log 2}{27}-\frac{N_{f}\left(132 \gamma_{E}+155-132 \log 2\right)}{27 N}\right. \\
&\left.+\frac{4 N_{f}^{2}\left(3 \gamma_{E}+1-3 \log 2\right)}{27 N^{2}}+\frac{N_{f}}{N^{3}}\right) \\
&= \frac{34 N^{3}-13 N^{2} N_{f}+3 N_{f}}{256 \pi^{4} N^{3}}=3 \beta_{1}
\end{aligned}
$$

Eq. (B.60) reads now:

$$
\begin{aligned}
g_{c d}^{2}(\mu) C_{1}^{(S)}(x)= & \frac{4 \beta_{0}}{\pi^{2} x^{4}} g_{c d}^{4}(\mu)\left(1+2 \beta_{0} g_{c d}^{2}(\mu) \log \left(x^{2} \mu^{2}\right)+g_{c d}^{4}(\mu)\left(3 \beta_{1} \log \left(x^{2} \mu^{2}\right)\right.\right. \\
& \left.\left.+3 \beta_{0}^{2} \log ^{2}\left(x^{2} \mu^{2}\right)\right)\right)
\end{aligned}
$$

In order to verify eq. (A.19) we insert the identity:

$$
1=\frac{1+\frac{\beta_{1}}{\beta_{0}} g_{c d}^{2}(\mu)}{1+\frac{\beta_{1}}{\beta_{0}} g_{c d}^{2}(x)} \frac{1+\frac{\beta_{1}}{\beta_{0}} g_{c d}^{2}(x)}{1+\frac{\beta_{1}}{\beta_{0}} g_{c d}^{2}(\mu)}
$$


in eq. (B.64) to the relevant perturbative order:

$$
\begin{aligned}
g_{c d}^{2}(\mu) C_{1}^{(S)}(x)= & \frac{1+\frac{\beta_{1}}{\beta_{0}} g_{c d}^{2}(\mu)}{1+\frac{\beta_{1}}{\beta_{0}} g_{c d}^{2}(x)} \frac{1+\frac{\beta_{1}}{\beta_{0}} g_{c d}^{2}(x)}{1+\frac{\beta_{1}}{\beta_{0}} g_{c d}^{2}(\mu)} \frac{4 \beta_{0} g_{c d}^{4}(\mu)}{\pi^{2} x^{4}} \\
& \left(1+g_{c d}^{2}(\mu) 2 \beta_{0} \log \left(x^{2} \mu^{2}\right)+g_{c d}^{4}(\mu)\left(3 \beta_{1} \log \left(x^{2} \mu^{2}\right)+3 \beta_{0}^{2} \log ^{2}\left(x^{2} \mu^{2}\right)\right)\right) \\
= & \frac{1+\frac{\beta_{1}}{\beta_{0}} g_{c d}^{2}(x)}{1+\frac{\beta_{1}}{\beta_{0}} g_{c d}^{2}(\mu)} \frac{4 \beta_{0} g_{c d}^{4}(\mu)}{\pi^{2} x^{4}}\left(1+g_{c d}^{2}(\mu) 2 \beta_{0} \log \left(x^{2} \mu^{2}\right)\right. \\
& \left.+g_{c d}^{4}(\mu)\left(2 \beta_{1} \log \left(x^{2} \mu^{2}\right)+3 \beta_{0}^{2} \log ^{2}\left(x^{2} \mu^{2}\right)\right)\right) \\
= & \frac{4 \beta_{0}}{\pi^{2} x^{4}} g_{c d}^{4}(x)\left(1+\frac{\beta_{1}}{\beta_{0}} g_{c d}^{2}(x)-\frac{\beta_{1}}{\beta_{0}} g_{c d}^{2}(\mu)\right)
\end{aligned}
$$

where we have expanded to the order of $g^{4}$ :

$$
\frac{1+\frac{\beta_{1}}{\beta_{0}} g_{a b}^{2}(\mu)}{1+\frac{\beta_{1}}{\beta_{0}} g_{a b}^{2}(x)}=1-\beta_{1} g_{a b}^{4}(\mu) \log \left(x^{2} \mu^{2}\right)
$$

and to the order of $g^{2}$ :

$$
\frac{1+\frac{\beta_{1}}{\beta_{0}} g_{a b}^{2}(x)}{1+\frac{\beta_{1}}{\beta_{0}} g_{a b}^{2}(\mu)}=1+\frac{\beta_{1}}{\beta_{0}} g_{a b}^{2}(x)-\frac{\beta_{1}}{\beta_{0}} g_{a b}^{2}(\mu)
$$

The factor of $\left(1+\frac{\beta_{1}}{\beta_{0}} g_{c d}^{2}(x)-\frac{\beta_{1}}{\beta_{0}} g_{c d}^{2}(\mu)\right)$ in the last line of eq. (B.66) arises according to eqs. (A.12), as it is verified by setting $\gamma_{0}^{\left(F^{2}\right)}=2 \beta_{0}$ and $\gamma_{1}^{\left(F^{2}\right)}=4 \beta_{1}$ in eq. (A.12):

$$
\begin{aligned}
& g_{c d}^{2}(\mu) C_{1}^{(S)}(x) \sim \frac{4 \beta_{0}}{\pi^{2} x^{4}} g_{c d}^{2}(x) g_{c d}^{2}(\mu) Z^{\left(F^{2}\right)}\left(x \mu, g_{c d}(\mu)\right) \\
& \sim \frac{4 \beta_{0}}{\pi^{2} x^{4}} g_{c d}^{2}(x) g_{c d}^{2}(\mu)\left(\frac{g_{c d}(x)}{g_{c d}(\mu)}\right)^{\frac{\gamma_{0}^{\left(F^{2}\right)}}{\beta_{0}}} \exp \left(\frac{\gamma_{1}^{\left(F^{2}\right)} \beta_{0}-\gamma_{0}^{\left(F^{2}\right)} \beta_{1}}{2 \beta_{0}^{2}}\left(g_{c d}^{2}(x)-g_{c d}^{2}(\mu)\right)+\cdots\right) \\
& \sim \frac{4 \beta_{0}}{\pi^{2} x^{4}} g_{c d}^{4}(x)\left(1+\frac{\beta_{1}}{\beta_{0}} g_{c d}^{2}(x)-\frac{\beta_{1}}{\beta_{0}} g_{c d}^{2}(\mu)+\cdots\right)
\end{aligned}
$$

Therefore, eq. (B.66) agrees with eq. (A.19).

We may remove the scale-dependent term, $-\frac{\beta_{1}}{\beta_{0}} g_{c d}^{2}(\mu)$, in the last line of eqs. (B.66) and (B.69) multiplying them by $\beta_{0}\left(1+\frac{\beta_{1}}{\beta_{0}} g_{c d}^{2}(\mu)\right)$ that makes $g_{c d}^{2}(\mu) C_{1}^{(S)}$ RG invariant to the relevant perturbative order. Hence, we get:

$$
-\frac{\beta(g)}{g} C_{1}^{(S)}(x) \sim \frac{4 \beta_{0}^{2}}{\pi^{2} x^{4}} g_{c d}^{4}(x)\left(1+\frac{\beta_{1}}{\beta_{0}} g_{c d}^{2}(x)\right) \sim \frac{4 \beta_{0}^{2}}{\pi^{2} x^{4}} g_{c d}^{4}(x)
$$

according to the nonperturbative asymptotics in eq. (5.2).

Open Access. This article is distributed under the terms of the Creative Commons Attribution License (CC-BY 4.0), which permits any use, distribution and reproduction in any medium, provided the original author(s) and source are credited. 


\section{References}

[1] M. Bochicchio, The large- $N$ Yang-Mills S-matrix is ultraviolet finite, but the large- $N$ QCD S-matrix is only renormalizable, Phys. Rev. D 95 (2017) 054010 [arXiv:1701.07833] [INSPIRE].

[2] V.A. Novikov, M.A. Shifman, A.I. Vainshtein and V.I. Zakharov, Are All Hadrons Alike?, Nucl. Phys. B 191 (1981) 301 [InSPIRE].

[3] M. Bochicchio, Renormalization in large-N QCD is incompatible with open/closed string duality, Phys. Lett. B 783 (2018) 341 [arXiv:1703.10176] [INSPIRE].

[4] J. Khoury and H.L. Verlinde, On open/closed string duality, Adv. Theor. Math. Phys. 3 (1999) 1893 [hep-th/0001056] [inSPIRE].

[5] P. Di Vecchia, A. Liccardo, R. Marotta and F. Pezzella, On the gauge/gravity correspondence and the open/closed string duality, Int. J. Mod. Phys. A 20 (2005) 4699 [hep-th/0503156] [INSPIRE].

[6] G. 't Hooft, A Planar Diagram Theory for Strong Interactions, Nucl. Phys. B 72 (1974) 461 [INSPIRE].

[7] G. Veneziano, Some Aspects of a Unified Approach to Gauge, Dual and Gribov Theories, Nucl. Phys. B 117 (1976) 519 [inSPIRE].

[8] G. Veneziano, An Introduction to Dual Models of Strong Interactions and Their Physical Motivations, Phys. Rept. 9 (1974) 199 [InSPIRE].

[9] O. Aharony, S.S. Gubser, J.M. Maldacena, H. Ooguri and Y. Oz, Large N field theories, string theory and gravity, Phys. Rept. 323 (2000) 183 [hep-th/9905111] [INSPIRE].

[10] M. Bochicchio, Open/closed string duality is incompatible with renormalization in 't Hooft large-N QCD, but not in Veneziano large-N QCD, PoS(EPS-HEP2017) 539 (2017).

[11] M.F. Zoller and K.G. Chetyrkin, OPE of the energy-momentum tensor correlator in massless QCD, JHEP 12 (2012) 119 [arXiv:1209.1516] [INSPIRE].

[12] M.F. Zoller, OPE of the energy-momentum tensor correlator and the gluon condensate operator in massless QCD to three-loop order, JHEP 10 (2014) 169 [arXiv:1407.6921] [INSPIRE].

[13] M.F. Zoller, On the renormalization of operator products: the scalar gluonic case, JHEP 04 (2016) 165 [arXiv: 1601.08094] [INSPIRE].

[14] A.L. Kataev, N.V. Krasnikov and A.A. Pivovarov, Two Loop Calculations for the Propagators of Gluonic Currents, Nucl. Phys. B 198 (1982) 508 [Erratum ibid. B 490 (1997) 505] [hep-ph/9612326] [INSPIRE].

[15] M. Bochicchio and S.P. Muscinelli, Ultraviolet asymptotics of glueball propagators, JHEP 08 (2013) 064 [arXiv: 1304.6409] [INSPIRE].

[16] M. Bochicchio, Glueball and meson propagators of any spin in large-N QCD, Nucl. Phys. B 875 (2013) 621 [arXiv: 1305.0273] [INSPIRE].

[17] V. Prochazka and R. Zwicky, Finiteness of two- and three-point functions and the renormalization group, Phys. Rev. D 95 (2017) 065027 [arXiv: 1611.01367] [INSPIRE].

[18] N. Arkani-Hamed and H. Murayama, Holomorphy, rescaling anomalies and exact $\beta$-functions in supersymmetric gauge theories, JHEP 06 (2000) 030 [hep-th/9707133] [INSPIRE]. 
[19] S.D. Joglekar and B.W. Lee, General Theory of Renormalization of Gauge Invariant Operators, Annals Phys. 97 (1976) 160 [INSPIRE].

[20] M. Henneaux, Remarks on the renormalization of gauge invariant operators in Yang-Mills theory, Phys. Lett. B 313 (1993) 35 [Erratum ibid. B 316 (1993) 633] [hep-th/9306101] [INSPIRE].

[21] J.C. Collins and R.J. Scalise, The Renormalization of composite operators in Yang-Mills theories using general covariant gauge, Phys. Rev. D 50 (1994) 4117 [hep-ph/9403231] [INSPIRE].

[22] V.P. Spiridonov, Anomalous Dimension of $G_{\mu \nu}^{2}$ and $\beta$-function, IYaI-P-0378 (1984) [INSPIRE].

[23] H. Simma, Equations of motion for effective Lagrangians and penguins in rare $B$ decays, Z. Phys. C 61 (1994) 67 [hep-ph/9307274] [INSPIRE].

[24] V.M. Braun, G.P. Korchemsky and D. Mueller, The Uses of conformal symmetry in QCD, Prog. Part. Nucl. Phys. 51 (2003) 311 [hep-ph/0306057] [INSPIRE].

[25] M. Bochicchio, An asymptotic solution of large- $N Q C D$ and of large- $N \mathcal{N}=1 S U S Y Y M$, in Prospects and Precision at the Large Hadron Collider at $14 \mathrm{TeV}$, Arcetri, Florence, Italy, September 1-October 24, 2014 (2014) [arXiv: 1409.5149] [INSPIRE].

[26] M. Becchetti, M. Bochicchio, Operator mixing and asymptotics in asymptotically free and logarithmic conformal theories, to appear in arXiv.

[27] J. Holland and S. Hollands, Recursive construction of operator product expansion coefficients, Commun. Math. Phys. 336 (2015) 1555 [arXiv:1401.3144] [INSPIRE].

[28] M.B. Fröb and J. Holland, All-order existence of and recursion relations for the operator product expansion in Yang-Mills theory, arXiv:1603.08012 [INSPIRE]. 